\title{
Coherent elastic neutrino-nucleus scattering in multi-ton scale dark matter experiments: classification of vector and scalar interactions new physics signals
}

\author{
D. Aristizabal Sierra, ${ }^{a, b}$ Bhaskar Dutta, $^{c}$ Shu Liao ${ }^{c}$ and Louis E. Strigari ${ }^{c}$ \\ ${ }^{a}$ Departamento de Física, Universidad Técnica Federico Santa María, \\ Casilla 110-V, Avda. España 1680, Valparaíso, Chile \\ ${ }^{b}$ IFPA, Dep. AGO, Université de Liège, \\ Bat B5, Sart Tilman B-4000 Liège 1, Belgium \\ ${ }^{c}$ Department of Physics and Astronomy, \\ Mitchell Institute for Fundamental Physics and Astronomy, Texas A\&M University, \\ College Station, TX 7r843, U.S.A. \\ E-mail: daristizabal@ulg.ac.be, dutta@physics.tamu.edu, \\ ikaros@physics.tamu.edu, strigari@physics.tamu.edu
}

ABSTRACT: We classify new physics signals in coherent elastic neutrino-nucleus scattering $(\mathrm{CE} \nu \mathrm{NS})$ processes induced by ${ }^{8} \mathrm{~B}$ solar neutrinos in multi-ton xenon dark matter (DM) detectors. Our analysis focuses on vector and scalar interactions in the effective and light mediator limits after considering the constraints emerging from the recent COHERENT data and neutrino masses. In both cases we identify a region where measurements of the event spectrum alone suffice to establish whether the new physics signal is related with vector or scalar couplings. We identify as well a region where measurements of the recoil spectrum are required so to establish the nature of the new interaction, and categorize the spectral features that enable distinguishing the vector from the scalar case. We demonstrate that measurements of the isospin nature of the new interaction and thereby removal of isospin related degeneracies are possible by combining independent measurements from two different detectors. We also comment on the status of searches for vector and scalar interactions for on-going multi-ton year xenon experiments.

Keywords: Beyond Standard Model, Neutrino Physics

ARXIV EPRINT: 1910.12437 


\section{Contents}

1 Introduction 1

2 Vector and scalar neutrino generalized interactions 3

2.1 Recoil spectrum and event rate 4

2.2 Constraints on vector and scalar couplings 5

2.2.1 Laboratory limits 5

2.2.2 Limits from neutrino scattering: $\mathrm{CE} \nu \mathrm{NS} \quad 6$

2.2.3 Limits on scalar interactions from neutrino masses 8

$\begin{array}{lll}2.2 .4 & \text { Astrophysical limits } & 10\end{array}$

3 Vector and scalar signals $\quad 11$

$\begin{array}{ll}3.1 \text { Light mediators } & 11\end{array}$

$\begin{array}{ll}3.2 & \text { Effective interactions } \\ \end{array}$

4 Isospin conserving versus isospin violating interactions $\quad \mathbf{1 5}$

$\begin{array}{ll}4.1 \text { The case of degeneracies } & 17\end{array}$

$5 \quad \mathrm{CE} \nu \mathrm{NS}$ and new interactions in XENON1T and LZ 19

6 Conclusions 20

\section{Introduction}

Next-generation direct detection dark matter (DM) experiments will be challenged by irreducible solar neutrino backgrounds and eventually, as exposure increases, by atmospheric neutrino fluxes as well [1]. With fairly large portions of the WIMP parameter space already explored, further exploration of DM direct detection signals in the near future call for multi-ton size detectors. Experiments such as XENONnT, LZ and DARWIN [2-4] soon after their operation will start observing neutrino-induced nuclear recoils, thus making the identification of an actual signal a difficult task. For that reason, experimental techniques that enable identifying background signal events from WIMP-induced recoils have been recently discussed. They include identification of WIMP signals by their direction dependencies $[5,6]$, time dependencies induced by WIMP and solar neutrino annual modulation [7] and multiple target detectors [8].

Although neutrino backgrounds certainly pose a problem for DM searches, they offer as well various physics opportunities, as they can be used as a tool for detailed studies of: (i) The coherent elastic neutrino-nucleus scattering ( $\mathrm{CE} \nu \mathrm{NS}$ ) process, (ii) solar and low-energy atmospheric neutrino fluxes, (iii) solar and supernova physics. Various analyses touching different aspects of these subjects have been already considered in the literature. 
Measurements of the $\mathrm{CE} \nu \mathrm{NS}$ process in DM detectors will provide complementary information to that arising from dedicated $\mathrm{CE} \nu \mathrm{NS}$ experiments such as COHERENT $[9,10]$, CONNIE [11], CONUS [12] and $\nu$-cleus [13]. That information can be used to test the presence of new physics in the form of e.g. neutrino non-standard interactions (NSI) [14-16], vector or scalar light mediators [17] or neutrino generalized interactions (NGI) [18]. The observation of the $\mathrm{CE} \nu \mathrm{NS}$ process will allow a better understanding of solar and atmospheric neutrino fluxes, the latter poorly understood with uncertainties of up to order $50 \%$. Precise measurements of low-energy solar neutrino fluxes will in turn improve upon our understanding of solar physics [19], while a ton-size detector such as XENONnT will be sensitive to a supernova burst up to $\sim 35 \mathrm{kpc}$ from earth, thus providing valuable information on supernova properties [20].

The $\mathrm{CE} \nu \mathrm{NS}$ and/or the electron-neutrino elastic cross sections are affected by the presence of new physics in different ways. Since neutrino-quark NSI are a parametrization of a four-fermion neutral current process, their effect is just a global rescaling (upwards or downwards) of the SM differential cross section [14, 15, 21]. Light mediators interactions (vector or scalar) change that behavior by introducing an extra momentum transfer dependence, which induces additional spectral features [21-27]. Neutrino electromagnetic couplings can potentially introduce spectral features as well [28-31]. Of particular interest are neutrino magnetic dipole moments which if sufficiently large lead to enhancements of the cross section at low recoil energies. NGI either in the light or effective limits have also different implications and depending on their nature lead to distinctive experimental signatures [18, 21, 26, 32-35]. Given the number of new physics scenarios and possible signatures that one could test through measurements of the CE $\nu$ NS process, it is desirable to systematically identify signatures that if observed could point towards the new physics responsible for a signal.

In this paper we consider such identification in the case of vector and scalar interactions in the light and effective regimes. For that aim we consider a xenon-based detector and $\mathrm{CE} \nu \mathrm{NS}$ induced by ${ }^{8} \mathrm{~B}$ solar neutrinos. We start by writing the interactions we are interested in section 2 and review the limits to which they are subject to in section 2.2. In section 2.2.2 we derive as well limits from neutrino masses that apply on scalar interactions (regardless of the size of the scalar mediator mass), and that arise through quark condensation contributing to either the Dirac or Majorana mass operators. Taking into account these limits, in particular those arising from COHERENT measurements, we then study the behavior of the signals according to their parameter space dependence in section 3. We first identify cases in which vector and scalar interactions can be distinguished by measurements of the event spectrum alone. We then identify cases in which combined measurements of the event and recoil spectrum are required. We evaluate as well the capability of multi-ton scale DM detectors to determine the isospin nature of the new physics signal (using silicon, argon and germanium in addition to xenon) in section 4 . We pay special attention to the case of degeneracies in xenon and determine the most suited nuclide for parameter degeneracy breaking in section 4.1. Finally in section 6 we present our conclusions. 


\section{Vector and scalar neutrino generalized interactions}

Vector and scalar NGI scenarios are dictated by the following interactions [26]

$$
\mathcal{L}^{V}=\bar{\nu} \gamma_{\mu}\left(f_{V}+i f_{A} \gamma_{5}\right) \nu V^{\mu}+\sum_{q=u, d} h_{V}^{q} \bar{q} \gamma_{\mu} q V^{\mu}
$$

and

$$
\begin{aligned}
& \mathcal{L}_{\mathrm{LNC}}^{S}=\bar{\nu}\left(f_{S}+i f_{P} \gamma_{5}\right) \nu S+\sum_{q=u, d} h_{S}^{q} \bar{q} q S, \\
& \mathcal{L}_{\mathrm{LNV}}^{S}=\overline{\nu^{c}}\left(f_{S}+i f_{P} \gamma_{5}\right) \nu S+\sum_{q=u, d} h_{S}^{q} \bar{q} q S,
\end{aligned}
$$

where in the scalar case lepton number conserving (LNC) couplings require the presence of right handed neutrinos. Note that the couplings can involve CP violating phases that we do not consider (see ref. [26] for an analysis including CP violation). The quark sector can involve as well axial and pseudoscalar currents. These couplings lead to nuclear spindependent processes which are suppressed compared with those induced by vector and scalar quark currents. The $\mathrm{CE} \nu \mathrm{NS}$ cross sections induced by the interactions in (2.1) and (2.2) are given by

$$
\begin{aligned}
\frac{d \sigma_{V}}{d E_{r}} & =\frac{G_{F}^{2}}{2 \pi} m_{N}\left|\xi_{V}\right|^{2}\left(2-\frac{E_{r} m_{N}}{E_{\nu}^{2}}\right) F^{2}\left(q^{2}\right), \\
\frac{d \sigma_{S}}{d E_{r}} & =\frac{G_{F}^{2}}{2 \pi} m_{N}\left|\xi_{S}\right|^{2} \frac{E_{r} m_{N}}{2 E_{\nu}^{2}} F^{2}\left(q^{2}\right),
\end{aligned}
$$

where $E_{r}$ refers to nuclear recoil energy $\left(E_{r}^{\max } \simeq 2 E_{\nu}^{2} / m_{N}\right.$, with $E_{\nu}$ the ingoing neutrino energy). Note that here we have assumed the same nuclear form factor for protons and neutrons. Such a choice is accurate provided one assumes the root-mean-square (rms) radii of the neutron and proton distributions are equal. Possible deviations from this assumption - allowed by uncertainties on the rms radius of the neutron distribution - require the proton and neutron contributions to be weighted by their own from factors [36]. For our analysis we use the Helm form factor [37]. For the rms radii of the proton distributions of xenon, silicon and germanium we use the average $\left\langle r_{k}\right\rangle=\sum_{k} X_{k} r_{k}$, where $r_{k}$ refers to the rms radius of the $k$-th isotope [38]. ${ }^{1}$ The new physics couplings are encoded in $\xi_{V}$ and $\xi_{S}$ which read

$$
\begin{aligned}
& \xi_{V}=g_{V}+\frac{C_{V}^{N} F_{V}}{\sqrt{2} G_{F}\left(2 m_{N} E_{r}+m_{V}^{2}\right)}, \\
& \xi_{S}=\frac{C_{S}^{N} F_{S}}{G_{F}\left(2 m_{N} E_{r}+m_{S}^{2}\right)},
\end{aligned}
$$

\footnotetext{
${ }^{1}$ Choosing a different value will not sizably affect our results. For ${ }^{8} \mathrm{~B}$ neutrino energies, the form factor approaches 1 .
} 


\begin{tabular}{|c|c|c|c|c|c|c|c|c|c|c|c|c|c|c|}
\hline & \multicolumn{2}{|c|}{ Silicon } & \multicolumn{2}{|r|}{ Argon } & \multicolumn{4}{|c|}{ Germanium } & \multicolumn{6}{|c|}{ Xenon } \\
\hline \multirow{3}{*}{ Nuc } & ${ }^{28} \mathrm{Si}$ & $9.22 \cdot 10^{-1}$ & ${ }^{36} \mathrm{Ar}$ & $3.37 \cdot 10^{-3}$ & ${ }^{70} \mathrm{Ge}$ & $2.04 \cdot 10^{-1}$ & ${ }^{72} \mathrm{Ge}$ & $2.73 \cdot 10^{-1}$ & ${ }^{124} \mathrm{Xe}$ & $9.50 \cdot 10^{-4}$ & ${ }^{126} \mathrm{Xe}$ & $8.90 \cdot 10^{-4}$ & ${ }^{128} \mathrm{Xe}$ & $1.91 \cdot 10^{-2}$ \\
\hline & ${ }^{29} \mathrm{Si}$ & $4.68 \cdot 10^{-2}$ & ${ }^{38} \mathrm{Ar}$ & $6.32 \cdot 10^{-4}$ & ${ }^{73} \mathrm{Ge}$ & $7.76 \cdot 10^{-2}$ & ${ }^{74} \mathrm{Ge}$ & $3.67 \cdot 10^{-1}$ & ${ }^{129} \mathrm{Xe}$ & $2.64 \cdot 10^{-1}$ & ${ }^{130} \mathrm{Xe}$ & $4.07 \cdot 10^{-2}$ & ${ }^{131} \mathrm{Xe}$ & $2.12 \cdot 10^{-1}$ \\
\hline & ${ }^{30} \mathrm{Si}$ & $3.09 \cdot 10^{-2}$ & ${ }^{40} \mathrm{Ar}$ & $9.96 \cdot 10^{-1}$ & ${ }^{76} \mathrm{Ge}$ & $7.83 \cdot 10^{-2}$ & - & - & ${ }^{132} \mathrm{Xe}$ & $2.69 \cdot 10^{-1}$ & ${ }^{134} \mathrm{Xe}$ & $1.04 \cdot 10^{-1}$ & ${ }^{136} \mathrm{Xe}$ & $8.86 \cdot 10^{-2}$ \\
\hline$m_{N}\left[\mathrm{GeV} / \mathrm{c}^{2}\right]$ & & 26.16 & & 37.21 & \multicolumn{4}{|c|}{67.66} & \multicolumn{6}{|c|}{122.29} \\
\hline$A_{N}$ & & 28.10 & & 39.98 & \multicolumn{4}{|c|}{72.70} & \multicolumn{6}{|c|}{131.39} \\
\hline
\end{tabular}

Table 1. Silicon, argon, germanium and xenon stable isotopes along with their relative abundances. Nuclear mass and mass numbers are calculated by averaging over the relative abundance of each isotope.

with $F_{V}=f_{V}-i f_{A}, F_{S}=f_{S}-i f_{P}$. The neutrino-nucleus vector couplings $C_{V}^{N}$ and $g_{V}$ (SM contribution) as well as the neutrino-nucleus scalar parameter are obtained by going from the quark to the nucleus operators. They are written as [18]

$$
\begin{aligned}
C_{V}^{N} & =Z\left(2 h_{V}^{u}+h_{V}^{d}\right)+(A-Z)\left(h_{V}^{u}+2 h_{V}^{d}\right), \\
g_{V} & =Z\left(2 g_{V}^{u}+g_{V}^{d}\right)+(A-Z)\left(g_{V}^{u}+2 g_{V}^{d}\right), \\
C_{S}^{N} & =Z \sum_{q=u, d} h_{S}^{q} \frac{m_{p}}{m_{q}} f_{T_{q}}^{p}+(A-Z) \sum_{q=u, d} h_{S}^{q} \frac{m_{n}}{m_{q}} f_{T_{q}}^{n} .
\end{aligned}
$$

Here $g_{V}^{u}=1 / 2-4 / 3 \sin ^{2} \theta_{W}, g_{V}^{d}=-1 / 2+2 / 3 \sin ^{2} \theta_{W}$ and $\sin ^{2} \theta_{W}=0.231$ [39]. In (2.8) contributions from the strange and heavy quarks have been neglected. Values for the hadronic form factors $f_{T_{q}}^{p, n}$ are derived in chiral perturbation theory from measurements of the $\pi$-nucleon sigma term [40-42]. Updated values are given by [41]

$$
\begin{array}{ll}
f_{T_{u}}^{p}=(20.8 \pm 1.5) \times 10^{-3}, & f_{T_{d}}^{p}=(41.1 \pm 2.8) \times 10^{-3}, \\
f_{T_{u}}^{n}=(18.9 \pm 1.4) \times 10^{-3}, & f_{T_{d}}^{n}=(45.1 \pm 2.7) \times 10^{-3} .
\end{array}
$$

Note that the new physics couplings in (2.5) reduce to effective couplings for $m_{X}^{2} \gg 4 E_{\nu}^{2}$ $(X=V, S)$, which for $E_{\nu} \lesssim 100 \mathrm{MeV}$ - as required by coherence of the neutrino-nucleus elastic scattering process - means that for $m_{X} \gtrsim 10^{3} \mathrm{MeV} \mathrm{CE} \nu \mathrm{NS}$ induced by the interactions in (2.1) and (2.2) is well described by the four-point contact interactions studied in refs. [18, 21, 34] (effective NGI) with

$$
\xi_{V}^{\mathrm{BSM}} \rightarrow \frac{C_{V}^{N} F_{V}}{\sqrt{2} G_{F} m_{V}^{2}}, \quad \xi_{S} \rightarrow \frac{C_{S}^{N} F_{S}}{G_{F} m_{S}^{2}} .
$$

More precise numbers are given in the section 2.2.2.

\subsection{Recoil spectrum and event rate}

We will consider silicon, argon, germanium and xenon detectors. For silicon, germanium and xenon the event rate spectrum comprises contributions from all their stable isotopes (see table 1). For argon only the effects of ${ }^{40} \mathrm{Ar}$ are relevant, given that its relative abundance amounts to $99.6 \%$. In our analysis rather than using the contributions from all 
isotopes we assume a single contribution by fixing the nuclear mass and mass number according to $\left\langle m_{N}\right\rangle=\sum_{i} X_{i} m_{i}$ and $\langle A\rangle=\sum_{i} X_{i} A_{i}$. Here $m_{i}$ refers to the mass of the $i$-th isotope in $\mathrm{GeV} / \mathrm{c}^{2}, A_{i}$ to its mass number and $X_{i}$ to its relative abundance.

The recoil spectrum can then be written as

$$
\frac{d R}{d E_{r}}=\frac{N_{A}}{\langle A\rangle} \int_{E_{\nu}^{\min }}^{E_{\nu}^{\max }} \Phi\left(E_{\nu}\right) \frac{d \sigma}{d E_{r}} d E_{\nu}
$$

where $N_{A}=6.022 \times 10^{26} \mathrm{~kg}^{-1}$ and $\Phi\left(E_{\nu}\right)$ the neutrino flux. The lower integration limit is given by $E_{\nu}^{\min }=\sqrt{\left\langle m_{N}\right\rangle E_{r} / 2}$, while the upper limit by the kinematic endpoint of the corresponding neutrino spectrum. The total number of events follows from integration of the recoil spectrum

$$
N_{\text {events }}=\int_{E_{r}^{\min }}^{E_{r}^{\max }} \frac{d R}{d E_{r}} \mathcal{A}\left(E_{r}\right) d E_{r}
$$

where $\mathcal{A}\left(E_{r}\right)$ refers to the experimental acceptance. For a binned analysis limits of integration are determined by bin width according to $E_{r} \pm \Delta E_{r}$.

\subsection{Constraints on vector and scalar couplings}

In this section we first discuss laboratory limits and then constraints from COHERENT. We then discuss bounds for light mediator scenarios arising from astrophysical and cosmological observations, most of them subject to fairly large uncertainties. Particularly useful for our analysis are the bounds arising from COHERENT.

\subsubsection{Laboratory limits}

Laboratory limits include bounds from fixed target and beam dump experiments, rare charged lepton decays, accelerator and neutrino data. They have been recently analyzed in ref. [43]. Here we briefly summarize them and discuss why for interactions (2.1) and (2.2) they can easily be evaded. Apart from accelerator searches, these limits apply only in the case of light mediators due kinematic constraints. Light vector or scalar bosons, if light enough, can be produced in the collision of protons with fixed targets. Production of $V$ can proceed via Bremsstrahlung or via pion production and subsequent decay, $\pi^{0} \rightarrow \gamma+V$, while production of $S$ only through Bremsstrahlung. Limits derived from these types of experiments rely on charged lepton decay modes, which the interactions in (2.1) and (2.2) generate only at the one-loop order. These limits therefore can be safely ignored.

Limits from rare charged lepton decays proceed from muon and tau decays modes comprising $V$ or $S$ in the final state, such as $\mu^{+} \rightarrow e^{+} \nu_{e} \bar{\nu}_{\mu}+X(X=S, V)$. As in the case of fixed target and beam dump experiments, limits derived from these type of processes require the new state to decay to charged leptons and so in our case these bounds can be readily evaded. LHC limits arise from Drell-Yan production, Higgs and $D$ meson decays (ATLAS, CMS and LHCb [44]). At $e^{+} e^{-}$colliders (KLOE, BaBar and Belle-II [45-47]) through radiative return and heavy meson decays. Relevant to our case is only DrellYan production which applies for vector or scalar masses above $12 \mathrm{GeV}$. Detection is done by looking for opposite-charge lepton pairs [44, 48], and so again these bounds we 
can safely ignore. Same arguments apply for bounds derived from neutrino data, which include neutrino trident production [49-51], Borexino [52] and Texono [53, 54], and which require couplings of the vector or scalar to charged leptons. In summary, in some cases the interactions in eqs. (2.1) and (2.2) allow the production of the vector or scalar bosons. However since detection relies on charged lepton decay modes, laboratory constraints in our case are loop suppressed.

For heavy states, bounds from muon and tau lepton flavor-violating decay modes $(\mu-e$ conversion in nuclei, $\mu \rightarrow e \gamma$ and $\tau \rightarrow \rho \ell, \ell=e, \mu)$, contact interactions and violation of universality could severely constraint the available parameter space [34, 55]. This however requires couplings to charged leptons and so these bounds in our case can be evaded too.

\subsubsection{Limits from neutrino scattering: $\mathrm{CE} \nu \mathrm{NS}$}

In general neutrino scattering data can be used to search for new physics or otherwise to set limits on new interactions. Sensitivities depend - of course - on the quality of the available data as well as on the uncertainties that the neutrino- $\mathcal{N}$ cross section involves $(\mathcal{N}$ stands for nucleus or nucleon, depending on the incoming neutrino energy). The CE $\nu \mathrm{NS}$ energy domain is determined by the coherence condition $q \lesssim R_{N}^{-1}$, and depending on the target material it is roughly below $100 \mathrm{MeV}$. The quality of the COHERENT data combined with a cross section with relatively small nuclear uncertainties, ${ }^{2}$ makes $\mathrm{CE} \nu \mathrm{NS}$ a rather powerful tool.

Depending on the energy window and on the neutrino energy and mediator mass relative size, other neutrino scattering processes can play a rather important role for $E_{\nu} \gtrsim 0.1 \mathrm{GeV}$. For $E_{\nu} \simeq 0.1-20 \mathrm{GeV}$ a number of scattering processes are relevant. They include neutrino quasi-elastic scattering, neutral current elastic scattering, resonant single pion production and coherent pion production. Recent measurements of these processes involve data from MiniBooNE [56-58], NOMAD [59] and MINER $\nu \mathrm{A}$ [60], among others. Constraints on new physics from these processes however are nonexistent. The reason could be related with the fact that these cross sections are subject to relatively large nuclear effects uncertainties.

For $E_{\nu} \simeq 20-500 \mathrm{GeV}$, the domain of deep inelastic scattering (DIS), the neutrino interacts with a quark in the nucleon. Data comes from CHARM-II [49] and NuTeV [51] and the cross section is subject to relatively small uncertainties, compared to the previous processes. Bounds for vector interactions (NSI) in the limit $m_{V}^{2} \gg 2 E_{\nu} m_{N} x y$ ( $x$ refers to the Bjorken variable, $y$ to the inelasticity parameter and $m_{N}$ to the nucleon mass), have been derived in [61]. DIS limits are nonexistent for scalar interactions nor for vector interactions in the mass range $m_{V}^{2} \simeq 2 E_{\nu} m_{N} x y$.

Focusing then on $\mathrm{CE} \nu \mathrm{NS}$, constraints on scalar and vector interactions in the light and effective regimes have been derived in a series of papers using COHERENT data [18, 23, 24, 28]. Recently using the Chicago-3 quenching factor [62] refs. [29, 63] updated those limits. We, however, recalculated them keeping the original quenching factor, but follow

\footnotetext{
${ }^{2}$ Mainly dominated by the lack of experimental information on the root-mean-square radius of the neutron distribution [36].
} 

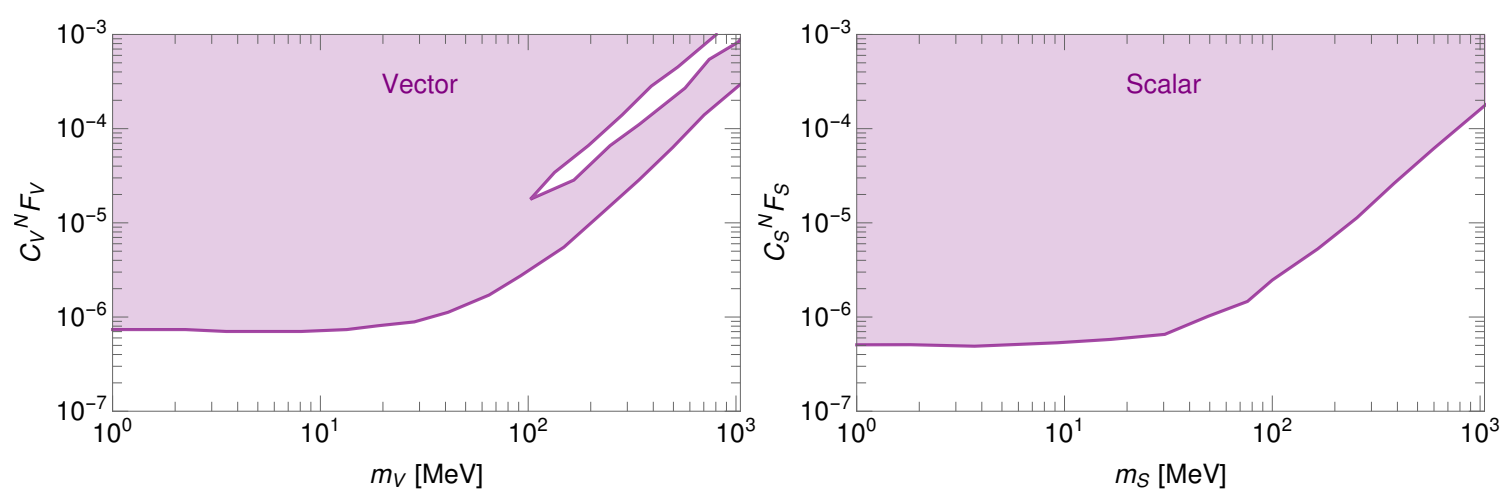

Figure 1. Left graph: $90 \%$ CL limits on vector couplings for light vector mediator scenarios from COHERENT data assuming an energy flat quenching factor [9], using spectral and time information and derived through a likelihood analysis. Right graph: same as left graph but for scalars.

an analyses which includes not only spectral information but temporal information as well $[64,65]$. And rather than adopting a chi-square test implement a likelihood statistical analysis (see below). The results are displayed in figure 1, which show the $90 \%$ CL limits in the $C_{X}^{N} F_{X}-m_{X}$ plane. In there one can see that at $m_{X} \gtrsim 30 \mathrm{MeV}$ the effective limit starts kicking in and at $m_{X} \simeq 10^{3} \mathrm{MeV} \mathrm{CE} \nu \mathrm{NS}$ is already dominated by it.

To derive these results we have used the spectral neutrino functions

$$
\begin{aligned}
& \mathcal{F}_{\nu_{\mu}}\left(E_{\nu}\right)=\frac{2 m_{\pi}}{m_{\pi}^{2}-m_{\mu}^{2}} \delta\left(1-\frac{2 E_{\nu}}{m_{\pi}^{2}-m_{\mu}^{2}}\right), \\
& \mathcal{F}_{\nu_{e}}\left(E_{\nu}\right)=\frac{192}{m_{\mu}}\left(\frac{E_{\nu}}{m_{\nu}}\right)^{2}\left(\frac{1}{2}-\frac{E_{\nu}}{m_{\mu}}\right), \\
& \mathcal{F}_{\bar{\nu}_{\mu}}\left(E_{\nu}\right)=\frac{64}{m_{\mu}}\left(\frac{E_{\nu}}{m_{\nu}}\right)^{2}\left(\frac{3}{4}-\frac{E_{\nu}}{m_{\mu}}\right),
\end{aligned}
$$

which are then normalized to $\mathcal{N}=r n_{\mathrm{POT}} /\left(4 \pi L^{2}\right)$, with $r=0.08, n_{\mathrm{POT}}=1.76 \times 10^{23}$ and $L=19.3 \mathrm{~m}$. Recoil energy binning is determined by number of photoelectrons $n_{\mathrm{PE}}$ which are related with $E_{r}$ through $n_{\mathrm{PE}}=1.17\left(E_{r} / \mathrm{keV}\right)[9,10]$. For the data analysis we define our likelihood function as:

$$
\mathcal{L}\left(\vec{\theta} \mid t, E_{r}\right)=\mathfrak{N} \prod_{\left(t, E_{r}\right)} \int d \alpha \sum_{N_{\mathrm{bg}}} P\left(N_{\mathrm{obs}}, \lambda\right) P\left(N_{\mathrm{obs}, \mathrm{bg}}, N_{\mathrm{bg}}\right) G\left(\alpha, \sigma_{\alpha}^{2}\right),
$$

where $P(n, \nu)=\nu^{n} e^{-\nu} / n$ ! refers to a Poisson distribution function, while $G\left(x, \sigma^{2}\right)=$ $e^{-x^{2} / 2 \sigma^{2}} / \sqrt{2 \pi \sigma^{2}}$ to a Gaussian distribution function with zero mean. The new physics parameters are encoded in the parameter space vector $\vec{\theta}, \mathfrak{N}$ is a normalization factor that assures unit normalization of the likelihood function when integrated over $\vec{\theta}, \lambda\left(t, E_{r}\right)=$ $(1+\alpha) N\left(t, E_{r}, \vec{\theta}\right)+N_{\mathrm{bg}}\left(t, E_{r}\right)$, is the expected/observed number of events with uncertainty parameter $\alpha$ accounting for the systematic uncertainties from flux, nuclear form factor, quenching factor and signal acceptance. From our notation in (2.14) it is clear that we assume this parameter follows a Gaussian distribution with zero mean and standard deviation $\sigma_{\alpha}=0.28[9] . N\left(t, E_{r}, \vec{\theta}\right)$ is the number of neutrino-induced recoil events 
predicted by theory and derived from (2.12) using eqs. (2.3) and (2.4). $N_{\mathrm{bg}}\left(t, E_{r}\right)$ is the true background count (not observed by definition), while $N_{\mathrm{obs}, \mathrm{bg}}\left(t, E_{r}\right)$ is the observed background reported by the COHERENT experiment. We therefore sum over $N_{\mathrm{bg}}\left(t, E_{r}\right)$ assuming a descrete flat prior distribution. We consider both energy and timing spectra by binning the data with 2 photoelectrons in recoil energy space and $0.5 \mu s$ in time space. Further details of this analysis can be found for the vector case in ref. [64].

Maximal enhancement of vector and scalar interactions happen for $m_{X}=1 \mathrm{MeV}$. For that value the exclusion plots in figures 1 fix the nuclear and neutrino couplings to $\left.C_{V}^{N} F_{V}\right|_{\operatorname{Exp}} \leq 7.4 \times 10^{-7}$ and $\left.C_{S}^{N} F_{S}\right|_{\operatorname{Exp}} \leq 5.1 \times 10^{-7}$. The value of the nuclear coupling depends on the nuclear target. For ${ }^{133} \mathrm{Cs}$, for which the fit has been done, one has

$$
\begin{aligned}
& C_{V}^{N} F_{V}=\left(211 h_{V}^{d}+188 h_{V}^{u}\right) F_{V}, \\
& C_{S}^{N} F_{S}=\left(1154.54 h_{S}^{d}+1117.53 h_{S}^{u}\right) F_{S} .
\end{aligned}
$$

Assuming $h_{X}^{q}=h_{X}$, i.e. assuming isospin conserving (violating) vector (scalar) interactions, bounds on the fundamental couplings read $h_{V} \times F_{V} \leq 2.0 \times 10^{-9}$ and $h_{S} \times F_{S} \leq 2.2 \times 10^{-10}$. Larger values are possible if a certain degree of fine tuning is at work, but we will not consider such possibility. With these numbers it becomes clear that the lighter (heavier) the isotope the less (more) prominent the effects, with the suppression (enhancement) given by $A / A_{\mathrm{Cs}}$.

We calculate the limits in the effective couplings at 90\% CL using COHERENT data with both temporal and energy spectrum $\left(\tilde{\xi}_{X}\right.$ refers to the numerical allowed values of the $\xi_{X}$ parameters in eq. (2.10)):

$$
\begin{aligned}
& \widetilde{\xi}_{V}=[1.06,13.01] \oplus[64.81,74.77], \\
& \widetilde{\xi}_{S}=[-16.08,16.08] .
\end{aligned}
$$

Notice that the $90 \%$ CL for effective couplings does not contains SM $\left(\widetilde{\xi}_{V}=0\right)$, this is because including temporal information of the COHERENT data gives signal of non-standard interaction at about $2 \sigma$ level as discovered in ref. [64].

\subsubsection{Limits on scalar interactions from neutrino masses}

Scalar interactions are subject as well to constraints from neutrino masses. Below $\Lambda_{\mathrm{QCD}} \simeq 200 \mathrm{MeV}$ quark condensates $\langle\bar{q} q\rangle$ induce a contribution to neutrino masses as depicted in figure 2. Since the neutrino mass operator is calculated at $m_{\nu}=\Sigma\left(p^{2}=0\right)$, the contribution is relevant regardless of the scalar mass. The overall mass scale of the neutrino mass matrix is determined by $G_{F}\langle\bar{q} q\rangle$, whereas the lepton flavor structure by the couplings $F_{S}$. The quark condensate can be evaluated in the Nambu-Jona-Lasinio model which gives $\langle\bar{q} q\rangle=(8 \pi / \sqrt{3}) f_{\pi}$ [66], with the value of the pion decay constant given by $f_{\pi}=89.8 \mathrm{MeV}$ as measured from the charged pion decay lifetime [67]. The neutrino mass contribution from the diagram in figure 2 can thus be written as

$$
m_{\nu}=\frac{8 \pi}{\sqrt{3}} G_{F} f_{\pi} F_{S} \sum_{q} h_{S}^{q} \simeq 122.5 \mathrm{eV} F_{S} \sum_{q} h_{S}^{q} .
$$




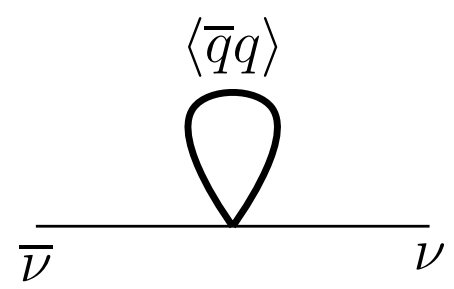

Figure 2. Contribution to neutrino masses from quark condensates induced by scalar interactions. The contribution can be of Dirac or Majorana type depending on whether the scalar coupling is or not lepton number violating. The loop refers to quark condensates. It is just a diagrammatic representation and so no loop suppression factor is involved.

At the $95 \% \mathrm{CL}$ cosmological limits on neutrino masses vary from $\sum m_{\nu}<0.6 \mathrm{eV}$ to $\sum m_{\nu}<0.12 \mathrm{eV}$ depending on the data sets used. The most stringent bound is obtained by including baryon acoustic oscillation data [68]. This limit combined with (2.17) implies

$$
F_{S} \times \sum_{q} h_{S}^{q}<9.79 \times 10^{-4} \quad \text { (neutrino mass limit) } .
$$

It can be satisfied with large $h_{S}^{q}$ and suppressed $F_{S}$ or vice versa. Large values for $F_{S}$ and $h_{S}^{q}$ are possible too, but a delicate cancellation between $h_{S}^{u}$ and $h_{S}^{d}$ is required. As discussed in the previous section, for $m_{S} \lesssim 10^{3} \mathrm{MeV}$ COHERENT constraints are more competitive. Indeed given the values that the couplings can have in that mass window, scalar interactions through quark condensates cannot sizably contribute to $\sum m_{\nu}$. Thus, if a signal of this type of interactions is observed and one can establish $m_{S} \lesssim 10^{3} \mathrm{MeV}$, one can be sure that neutrino mass generation should proceed through a different mechanism.

For $m_{S} \gtrsim 10^{3} \mathrm{MeV}$ one is already in the effective limit where scalar interactions are controlled by the scalar parameter in (2.10). In terms of the quark couplings this parameter is maximized in the limit $h_{S}^{u} \rightarrow h_{S}^{d} \rightarrow h_{S}$ for which, using the central values of the hadron form factors in (2.9), $C_{S}^{N}$ can be written as

$$
C_{S}^{N}=h_{S}[16.54(A-Z)-16.58 Z] .
$$

Thus, combined with the neutrino mass limit the strength of the scalar interaction compared with the SM contribution is bounded as follows

$$
\begin{aligned}
& \mathrm{Xe}: \xi_{S} \lesssim\left(\frac{302.2}{m_{S} / \mathrm{GeV}}\right)^{2}, \quad \mathrm{Ge}: \xi_{S} \lesssim\left(\frac{224.8}{m_{S} / \mathrm{GeV}}\right)^{2}, \\
& \mathrm{Ar}: \xi_{S} \lesssim\left(\frac{166.6}{m_{S} / \mathrm{GeV}}\right)^{2}, \quad \mathrm{Si}: \xi_{S} \lesssim\left(\frac{139.8}{m_{S} / \mathrm{GeV}}\right)^{2} .
\end{aligned}
$$

As expected from (2.19) the limit is isotope dependent, but the differences between heavy (xenon), intermediate (germanium) and light (silicon and argon) nuclides is at most a factor $\sim 2$. The result in (2.20) demonstrates that only for $m_{S} \gtrsim 300 \mathrm{GeV}$ neutrino mass limits lead to suppressed scalar couplings. Indeed, they show that if one takes into account only that constraint the scalar interaction can be way larger than the SM contribution when $m_{S} \lesssim 300 \mathrm{GeV}$. 


\subsubsection{Astrophysical limits}

Scalar interactions are subject to further constraints that apply on either quark or neutrino couplings or both simultaneously. They were recently discussed in ref. [23] and below we summarize them. These limits can be sorted in three different groups depending on how they affect neutrino properties within the supernova (SN) inner core. Since neutrinos are trapped in the SN core they can only escape by diffusion, with the diffusion time determined by $t_{\mathrm{diff}} \simeq R_{\mathrm{SN}}^{2} / \lambda\left(R_{\mathrm{SN}}\right.$ is the SN core radius and $\lambda$ the neutrino mean free path). Regardless of whether the interaction is or not lepton number violating its presence can reduce $t_{\text {diff }}$ by increasing $\lambda$. Assuring that neutrino trapping is not strongly disrupted, i.e. that $t_{\text {diff }}$ does not decreases below $\sim 10$ secs, translates into an upper bound on scalar couplings that depends on the scalar mass, $C_{S}^{N} F_{S} \lesssim 1.2 \times 10^{-7}$ for $m_{S}=1 \mathrm{MeV}$ and about an order of magnitude larger for $m_{S}=100 \mathrm{MeV}$.

In the LNC case, more stringent bounds follow from SN cooling and sterile neutrino trapping, with the strongest bound arising from the former. Since sterile neutrinos are not subject to electroweak interactions, once they are produced they can escape the SN core, thus leading to fast energy loss if the active-sterile neutrino rate conversion is high enough. In the LNV case, instead, the new interaction can modify the electron neutrino chemical potential for sufficiently large couplings, thus affecting the SN equation of state; something that can be understood fully in terms of the electron neutrino chemical potential. In the absence of new interactions an electron neutrino asymmetry is present $\left(\mu_{\nu_{e}} \neq 0\right.$, with $\mu_{\nu_{e}}$ the electron neutrino chemical potential). If the LNV interaction attains thermal equilibrium it will enforce $4 \mu_{\nu_{e}}=\mu_{S}$, which implies $n_{\nu_{e}}=n_{\bar{\nu}_{e}}$ given that $\mu_{S}=0 .{ }^{3}$ In other words, if the new couplings are large enough the interaction will tend to equilibrate $n_{\nu_{e}}$ and $n_{\bar{\nu}_{e}}$, affecting the SN equation of state. The limits derived from these arguments depend on the scalar mass. For $m_{S}=1 \mathrm{MeV}$ in the LNC case the most stringent limit reads $C_{S}^{N} F_{S} \lesssim 3.3 \times 10^{-9}$, while for LNV interactions $C_{S}^{N} F_{S} \lesssim 2.6 \times 10^{-10}$.

Light vectors are subject to limits from stellar cooling and SN arguments as well. Since the temperature of the helium core in horizontal branch stars is of order $10^{8} \mathrm{~K} \simeq 10^{-2} \mathrm{MeV}$, vector bosons with masses up to $0.1 \mathrm{MeV}$ can be produced through ${ }^{4} \mathrm{He}$ Compton scattering processes (this value possible from the energy tail of the distribution). Avoiding energy loss through these processes implies $h_{V}^{p, n} \lesssim 4 \times 10^{-11}[69,70]$. Constraints from disruption of the neutrino diffusion time in SN apply as well [71].

There is however few caveats on these bounds that one should bear in mind. First of all, uncertainties on core-collapse SN are still large. Limits derived from SN arguments therefore should be understood as order of magnitude estimations [72]. For vectors, stellar cooling arguments ignore plasma mixing effects, considering them results in different bounds [73]. And finally, these limits can be avoided if the new states couple to scalars that condensate inside the star or the SN core. In that case the mass of the new states is proportional to the medium mass density, and so their production is no longer possible [74, 75]. In our analysis therefore we will not consider them.

\footnotetext{
${ }^{3}$ The generation of an asymmetry in $S$ (a chemical potential) requires $S \neq S^{*}$, departure from thermal equilibrium and a $\mathrm{CP}$-violating interaction.
} 


\section{Vector and scalar signals}

In this section we discuss features of both, number of events and recoil spectrum that can be used to differentiate vector from scalar interactions signals. The discussion is split in light and effective interactions. For the former we go into the "deep" light regime, which from figures 1 it can be seen corresponds to $m_{X} \lesssim 10 \mathrm{MeV}$. For the latter, instead, we assume $m_{X} \gtrsim 10^{3} \mathrm{MeV}$. As for the bounds, in the light mediator case we use limits from figures 1 while in the effective case we rather use bounds in (2.16). In both cases we use the ${ }^{8} \mathrm{~B}$ solar neutrino flux and xenon as target material. This choice is well motivated by xenon-based experiments such as XENONnT, LZ and DARWIN which will be subject to ${ }^{8} \mathrm{~B}$ neutrino backgrounds [2-4]. In order to be the less experiment dependent, we assume a ton-year exposure and $100 \%$ efficiency. For a given parameter choice results are expressed in terms of

$$
R \equiv \frac{N_{\mathrm{SM}+\mathrm{X}}}{N_{\mathrm{SM}}}
$$

where $N_{\mathrm{SM}}$ refers to the number of events as expected in the SM and depending on the case $X=V, S$.

\subsection{Light mediators}

For the light vector mediator case $C_{V}^{N} F_{V} \leq 7.4 \times 10^{-7}$, while for scalar $C_{S}^{N} F_{S} \leq 5.1 \times 10^{-7}$. Assuming universal quark couplings these bounds can be translated into bounds in xenon just by scaling by $A_{\mathrm{Xe}} / A_{\mathrm{Cs}} \simeq 0.99$. For the calculation of the recoil spectrum we vary $E_{r}$ from $10^{-1} \mathrm{keV}$ up to the recoil energy allowed by the neutrino energy tail of the ${ }^{8} \mathrm{~B}$ neutrino spectrum, $E_{\nu}=16.56 \mathrm{MeV}$ [76]. For the calculation of the event spectrum $N_{\text {events }}$ we use a $10^{-1} \mathrm{keV}$ threshold and integrate up to $E_{r}=97 \mathrm{keV} .{ }^{4}$ With that choice we get $N_{\text {events }}^{\mathrm{SM}}=760.25$.

The expected number of events (normalized to the SM expectation) as a function of $C_{X} F_{X}$ is shown in the left graph in figure 3. In there one can see that for small coupling, $C_{X}^{N} F_{X} \lesssim 10^{-9}$, the new interactions do not generate any sizable deviation above or below the SM expectation. Right after that value vector interactions start depleting the SM contribution, while effects of the scalar (which can only produce enhancements of the signal) start being visible only above $10^{-8}$. From that point on up to about an order of magnitude $\left(7 \times 10^{-8}\right)$ vector and scalar interactions behave rather differently, producing event rate spectra which have no overlapping at all. Thus in that coupling range, no matter the mediator mass as far as it is light, measurements can differentiate between them both. From that value and up to the value allowed by COHERENT constraints (vertical orange and purple lines for scalar and vector, respectively), three regions can be distinguished: region I where $R=N_{\mathrm{SM}+X} / N_{\mathrm{SM}} \gtrsim 84$, region II with $R \cong(1,84]$ and region III where $R<1$. Regions I and III are covered only by vector interactions, therefore a signal featuring such values will favor a light vector mediator over the scalar. Region II is problematic in the sense that in there using only measurements of $R$ one cannot tell the nature of the

\footnotetext{
${ }^{4}$ Note that since the vector interactions in (2.1) are flavor universal matter (propagation) effects factor out. For matter effects in lepton flavor-violating vector light mediator models see ref. [77].
} 

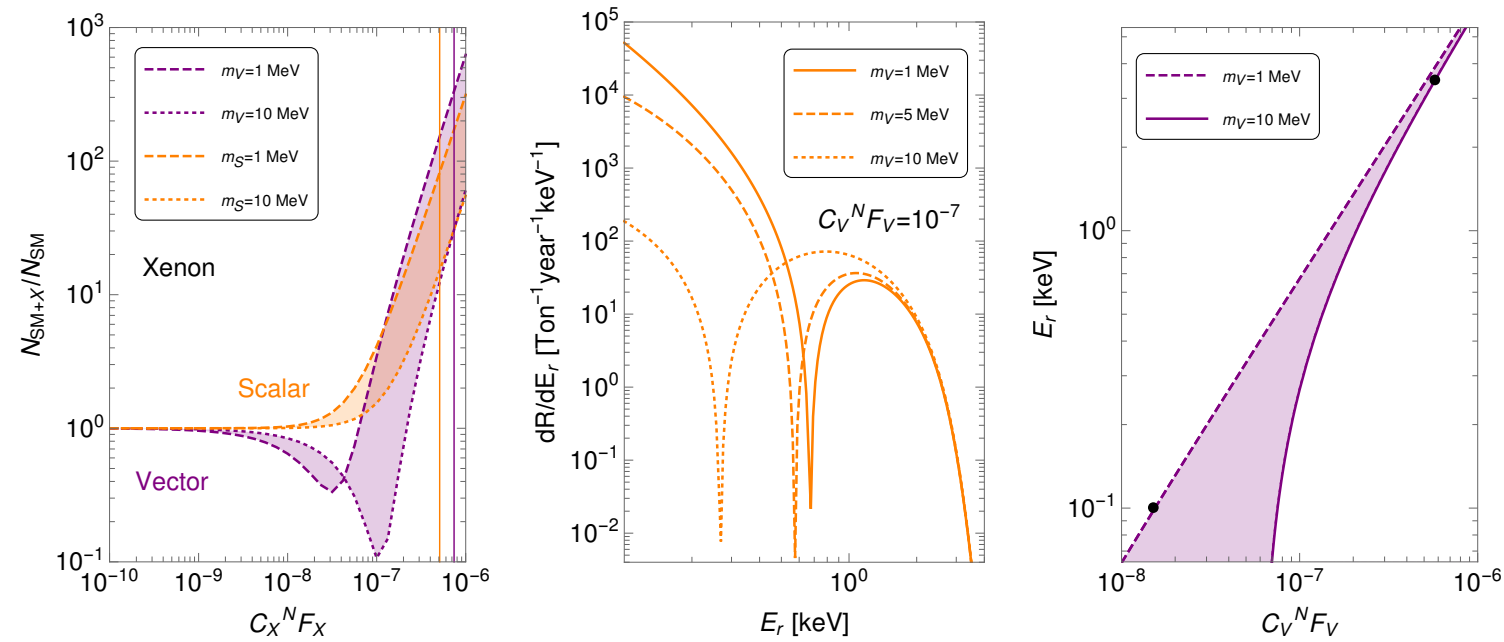

Figure 3. Left graph: number of expected events normalized to the SM expectation for light vector (purple) and scalar (orange) mediators in a xenon detector assuming a one ton-year exposure and $100 \%$ detector efficiency. The result was obtained assuming a $10^{-1} \mathrm{keV}$ energy recoil threshold, $X=V, S$. The vertical orange and purple lines indicate COHERENT upper limits for scalar and vector couplings, respectively. Middle graph: recoil spectrum for different light vector mediator masses as a function of recoil energy. This result demonstrates the presence of dips in the vector spectrum, in contrast to the scalar case. Right graph: energy recoil region where dips are found in the light vector mediator case. The black dots located at $\left(C_{V}^{N} F_{V}, E_{r}\right)=\left(1.5 \times 10^{-8}, 10^{-1} \mathrm{keV}\right)$ and $\left(C_{V}^{N} F_{V}, E_{r}\right)=\left(5.8 \times 10^{-7}, 3.5\right) \mathrm{keV}$ fix the boundaries where dips, though present, are no longer observable in the recoil spectrum. See text (section 3.1) for further details.

new contribution. There is however an interesting way through which measurements of $R$ combined with measurements of the recoil spectrum can provide a conclusive answer in almost all parameter space. Let us discuss this in more detail.

In contrast to scalar interactions vector can produce depletions below the SM expectation. And at the recoil spectrum level can lead to dips in the spectrum, as exemplified in the middle graph in figure 3 which shows the recoil spectrum for $m_{V}=1,5,10 \mathrm{MeV}$ calculated for $C_{V} F_{V}=10^{-7}$. For a given coupling $C_{V}^{N} F_{V}$ and vector boson mass $m_{V}$ the location of such dip is determined by the condition [26]

$$
E_{r}=\frac{C_{V}^{N} F_{V}-\sqrt{2} G_{F}\left|g_{V}\right| m_{V}^{2}}{2 \sqrt{2} G_{F}\left|g_{V}\right| m_{N}} .
$$

For the parameters that define region II and within the "deep" light vector mediator window we are considering this implies that those dips are located within the recoil energy interval $[0.46,4.9] \mathrm{keV}$, with the left boundary of the interval obtained for $\left(C_{V}^{N} F_{V}, m_{V}\right)=$ $\left(7 \times 10^{-8}, 1 \mathrm{MeV}\right)$ and the right for $\left(C_{V}^{N} F_{V}, m_{V}\right)=\left(7.4 \times 10^{-7}, 4 \mathrm{MeV}\right)$. Bearing in mind that the observable recoil energy window is defined by $\left[10^{-1}, 3.5\right] \mathrm{keV}$ (the value to the left determined by rather optimistic future thresholds, while the value to the right by the kinematic endpoint energy of the ${ }^{8} \mathrm{~B}$ neutrino spectrum $),{ }^{5}$ this means that some dips

\footnotetext{
${ }^{5}$ Note that choosing a less optimistic threshold, which very likely will be the case, does not change our conclusion: observation of a dip will discard scalar interactions as being responsible for the signal.
} 
are not observable. This happens for parameters in the range $C_{V}^{N} F_{V} \lesssim 1.5 \times 10^{-8}$ and $C_{V}^{N} F_{V} \gtrsim 5.8 \times 10^{-7}$, determined by the points where the boundaries $10^{-1} \mathrm{keV}$ and $3.5 \mathrm{keV}$ intercept the isocontours $m_{V}=1 \mathrm{MeV}$ and $m_{V}=10 \mathrm{MeV}$ in the right graph in figure 3, indicated by the black points. For $C_{V}^{N} F_{V} \lesssim 1.5 \times 10^{-8}$ the expected number of events barely exceeds the SM expectation, and so in that regard that region basically does not differ from the region for which $C_{V}^{N} F_{V} \lesssim 10^{-9}$. For $C_{V}^{N} F_{V} \gtrsim 5.8 \times 10^{-7}$, once is already in a region where COHERENT bounds on scalar interactions rules out the possibility of a signal from scalar couplings; and so differentiation is possible. In conclusion, combined measurements of $R$ and of the recoil spectrum will suffice - in principle - to determine the nature of the new physics in region II as well.

\subsection{Effective interactions}

For the effective vector mediator analysis we drop the $q^{2}$ dependence in (2.5) and write the coupling according to $\xi_{V}=g_{V}+\widetilde{\xi}_{V}$, with $\widetilde{\xi}_{V}$ subject to the constraints in (2.16). For the effective scalar calculation we drop as well the $q^{2}$ dependence in $\xi_{S}$ in (2.5) and treat $\xi_{S}$ as a free parameter subject to the limits in (2.16). By comparing the SM+vector and SM differential cross sections, eqs. (2.3), one can see that in the effective vector case deviations in the $\mathrm{CE} \nu \mathrm{NS}$ process are entirely controlled by the ratio $\xi_{V}^{2} / g_{V}^{2}$ [15]. In contrast scalar interactions have a different energy dependence, and so a full calculation of the recoil spectrum and its integration according to eq. (2.12) are required.

The left graph in figure 4 shows the expected number of events (normalized to the SM expectation) as a function of the effective couplings $\widetilde{\xi}_{X} \quad(X=V, S)$. The shadowed vertical stripes indicate the $90 \% \mathrm{CL}$ limits on $\xi_{V}$ (purple) and $\xi_{S}$ (orange). In terms of $R$ two regions can be identified. A region entirely dominated by the vector interaction where $R \lesssim 0.94$ (the new vector interaction destructively interferes with the SM contribution) and a second region where $R>1$. In the latter two subregions can be identified: (i) One where $R$ exceeds 1.05 but never above 1.08, (ii) a second where $R$ goes above the SM expectation but does not exceed 1.05. Measurements yielding $R \lesssim 0.94$ or $R \subset[1.05,1.08]$ will point to a vector interaction as responsible for the signal. In contrast, for measurements resulting in $R \subset(1,1.05], R$ alone cannot be used to disentangle whether the signal is related with a vector or a scalar interaction.

To break such "degeneracy" one can check the recoil energy spectrum and see whether vector and scalar interactions lead to different spectral features. The key observation here is that the differential cross sections for scalar and vector interactions have different recoil energy dependencies. As a consequence the effect of vector interactions is just an overall rescaling of the SM expectation, while for the scalar coupling energy dependent differences are found. This can be seen in the middle graph in figure 4 which shows the vector and scalar recoil spectra evaluated for couplings in that region: $\xi_{V}=74.18$ and $\left|\xi_{S}\right|=16.08$. One can see that scalar interactions produce recoil spectra that at small recoil energies tend to overlap with the SM expectation and to departure from it at higher $E_{r}$. This is not the case for vector-induced spectra which follow the SM spectrum up to a multiplicative factor. Disentanglement of vector and scalar interactions in the region $R \cong(1.0,1.05]$ can then be done - in principle - combining information on $R$ and on the recoil spectrum, 

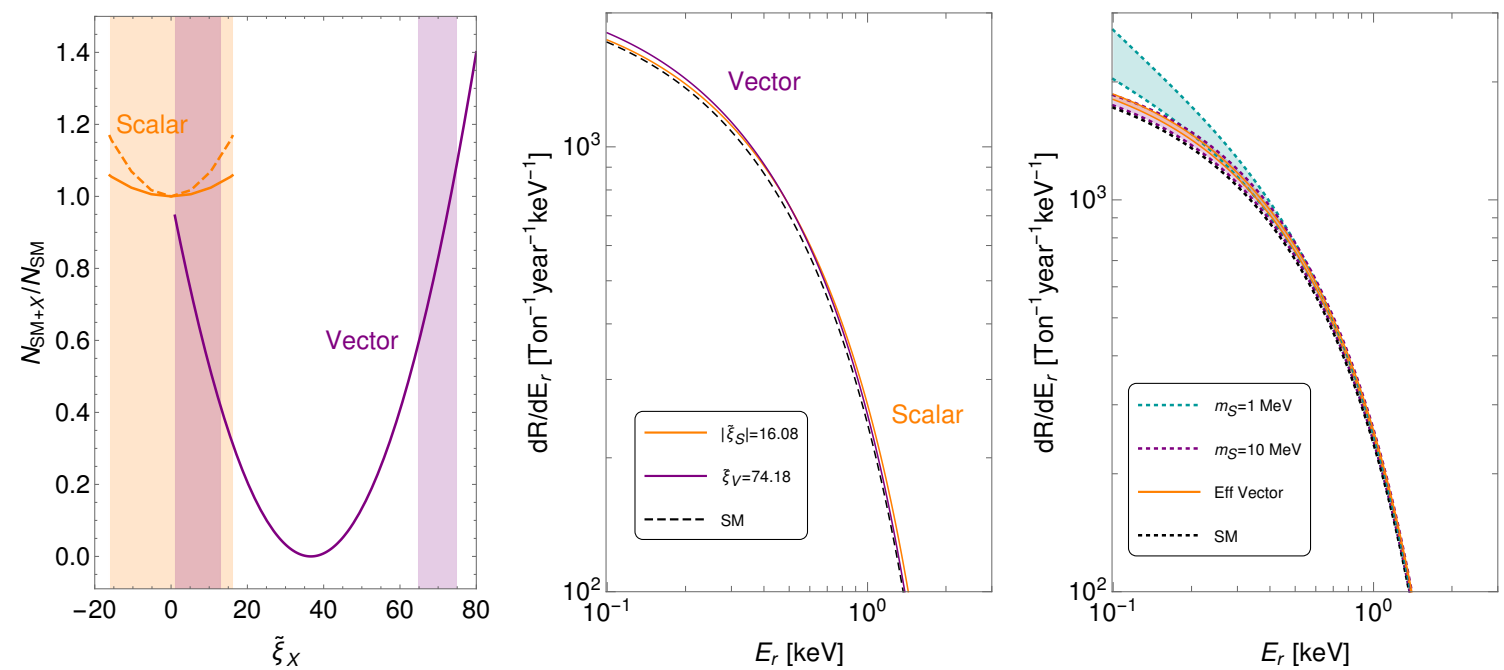

Figure 4. Left graph: expected number of events in the presence of vector and scalar effective interactions $\left(m_{X} \gtrsim 10^{3} \mathrm{MeV}\right)$ normalized to the SM expectation as a function of the effective parameter $\widetilde{\xi}_{X} \quad(X=V, S)$. The calculation is done assuming a $10^{-1} \mathrm{keV}$ threshold, a 1 tonyear exposure, $100 \%$ detector efficiency and xenon as target material. Included as well is the result for scalar interactions obtained by taking a $1 \mathrm{keV}$ threshold (dashed orange curve). The shadowed stripes indicate the $90 \%$ CL limits from COHERENT data. Middle graph: recoil spectra for which effective vector and scalar interactions cannot be differentiated by only measurements of $R \equiv N_{\mathrm{SM}+\mathrm{X}} / N_{\mathrm{SM}} \subset(1.0,1.05]$. This result demonstrates that measurements of $R$ combined with measurements of the recoil spectrum can be used to tell whether the signal is due to vector or scalar effective couplings. Right graph: comparison of recoil spectra for light scalar mediators and effective vector interactions. Integration of these recoil spectra lead to $R \subset[1.05,1.08]$ in both cases. Combined measurements of $R$ and the recoil spectrum can then be used to distinguish the interaction responsible for the signal. See text (section 3.2) for further details.

provided the new physics couplings have values such that sizable departures from the SM prediction are observed and the detector has a good spectral resolution.

Regarding the effective interactions analysis there is however a caveat on some of our conclusions. Measurements yielding $R \cong[1.05,1.08]$ can be obtained as well in the light scalar mediator case, as shown in the left graph in figure 3. So although such measurements cannot result from effective scalar interactions, they can if the scalar mediator is light. For a light scalar, $R \cong[1.05,1.08]$ results from $C_{S}^{N} F_{S} \subset[1.2,2.6] \times 10^{-8}$ for $m_{V}=1 \mathrm{MeV}$ or $C_{S}^{N} F_{S} \subset[1.5,3.7] \times 10^{-8}$ for $m_{V}=10 \mathrm{MeV}$. This ambiguity can be - in principle removed with the aid of the recoil spectrum. As shown in the right graph in figure 4, whether this is the case depends on $m_{S}$. For values of $m_{S}$ close to $1 \mathrm{MeV}$ the recoil spectrum is rather peaked at low energies and largely differs from the recoil spectra induced by effective vector interactions. As $m_{S}$ increases towards values close to $10 \mathrm{MeV}$ a strong overlapping between light and effective spectra is instead found. Thus the question of whether one can disentangle vector (effective) and scalar (light) interactions in the region $R \cong[1.05,1.08]$ depends to a large extent on $m_{S}$. 


\section{Isospin conserving versus isospin violating interactions}

In what follows we discuss the capability of ton-size DM detectors to identify the isospin nature of the new interaction. From eqs. (2.6) and (2.8) one can see that the conditions

$$
h_{V}^{u}=h_{V}^{d}, \quad h_{S}^{u}=11.9 h_{S}^{d},
$$

assure isospin conserving interactions (for the scalar case we are using the hadronic form factors central values, see eq. (2.9)). Deviations from these relations lead to isospin violation, of which one can distinguish three (extreme) particular cases. Protophobic and neutrophobic interactions and degeneracies. The latter defined as a region in parameter space for which the new physics signal exactly vanishes in a given detector (for a given nuclide). The relations between the fundamental quark couplings that define each case depend - of course - on whether the interaction is vector or scalar (regardless of the size of the mediator mass). Starting with the vector case one finds (degeneracy in a $\left(A_{1}, Z_{1}\right)$ nucleus):

$$
\begin{aligned}
\text { Protophobic: } & h_{V}^{u}=-h_{V}^{d} / 2, \\
\text { Neutrophobic: } & h_{V}^{u}=-2 h_{V}^{d}, \\
\text { Degeneracy : } & h_{V}^{u}=-\frac{2 A_{1}-Z_{1}}{A_{1}+Z_{1}} h_{V}^{d} .
\end{aligned}
$$

For scalar interactions instead the relations can be written as

$$
\begin{aligned}
\text { Protophobic : } \quad h_{S}^{u} & =-\frac{m_{u}}{m_{d}} \frac{f_{T_{d}}^{p}}{f_{T_{u}}^{p}} h_{S}^{d}=-0.92 h_{S}^{d}, \\
\text { Neutrophobic : } \quad h_{S}^{u} & =-\frac{m_{u}}{m_{d}} \frac{f_{T_{d}}^{n}}{f_{T_{u}}^{n}} h_{S}^{d}=-1.11 h_{S}^{d}, \\
\text { Degeneracy : } \quad h_{S}^{u} & =-\frac{m_{u}}{m_{d}} \frac{Z_{1} m_{p} f_{T_{d}}^{p}+N_{1} m_{n} f_{T_{d}}^{n}}{Z_{1} m_{p} f_{T_{u}}^{p}+N_{1} m_{n} f_{T_{u}}^{n}} h_{S}^{d} \\
& =-\frac{9.01 A_{1}-0.81 Z_{1}}{8.07 A_{1}+0.79 Z_{1}} h_{S}^{d} .
\end{aligned}
$$

Experimentally establishing isospin conservation or these isospin-violating scenarios (or any other intermediate isospin-violating case) cannot be done on the basis of a single measurement. In the case of degeneracies is rather obvious that at least two independent measurements are required. One in which no deviation from the SM is observed and one where degeneracy is broken, at least partially. In the protophobic and neutrophobic cases the reason as well is rather simple. The new physics is controlled by $C_{X}^{N} F_{X}$ which can be "reparametrized" according to

$$
\begin{aligned}
F_{V} C_{V}^{N} & =F_{V} h_{V}^{p}\left[Z+r_{V}(A-Z)\right], \\
F_{S} C_{S}^{N} & =F_{S} h_{S}^{p}\left[Z+r_{S}(A-Z)\right] .
\end{aligned}
$$

These quantities involve the overall factor and the ratio $r_{X}=h_{X}^{n} / h_{X}^{p}$, which a single measurement cannot fix without ambiguity. Two measurements in different detectors instead will enable pinning down their values, at least in the effective case. 

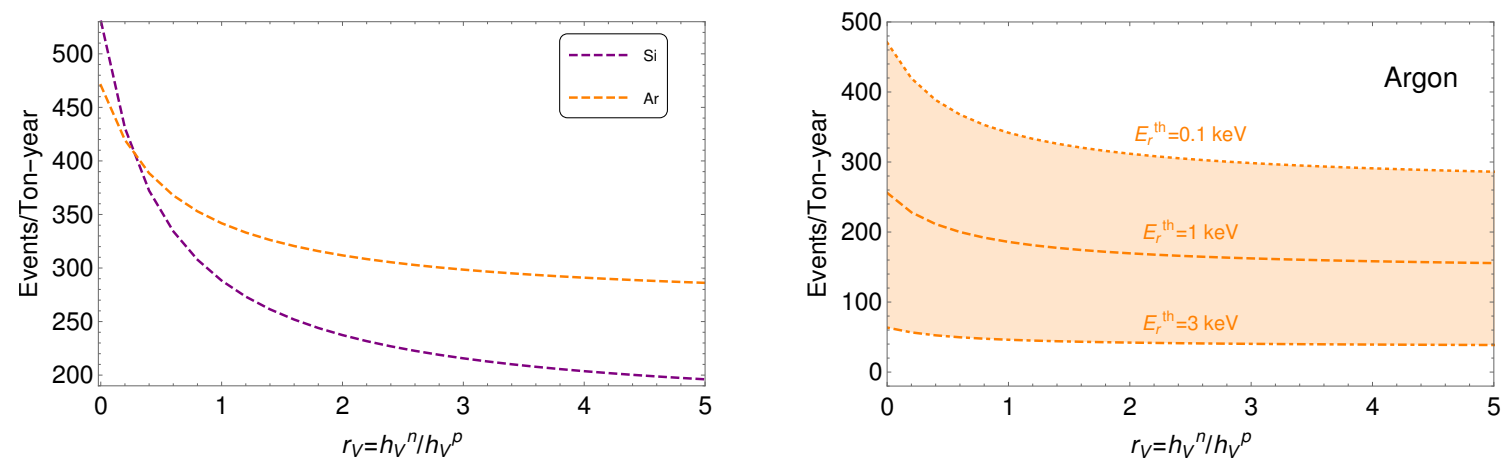

Figure 5. Left graph: expected number of events in silicon and argon detectors as a function of $r_{V}=h_{V}^{n} / h_{V}^{p}\left(h_{V}^{n}\right.$ and $h_{V}^{p}$ refer to the couplings of the new "heavy" vector to neutrons and protons respectively). The result was obtained assuming a xenon detector has measured $R=1.08$ (i.e. $N_{\text {events }}^{\mathrm{Xe}}=821$ events/ton-year) and an effective vector interaction has been established as responsible for the signal (see section 3.2). For this particular benchmark case, measurements $N_{\text {events }}^{\mathrm{Si}} \simeq 288$ and $N_{\text {events }}^{\mathrm{Ar}} \simeq 342$ will favor an isospin conserving interaction $\left(r_{V}=1\right)$, deviations from these values will instead favor isospin violation. Right graph: number of events/ton-year in an argon detector for various recoil energy thresholds. For $E_{r}^{\text {th }}>3 \mathrm{keV}$, the lack of statistics will make the isospin test analysis hard. In that case exposures above 1 ton-year will be required [78].

To demonstrate how this can be done we focus on the effective vector case and assume that out of the two different measurements one is done in a xenon-based detector. This means that a deviation in the SM prediction has been observed and that using the arguments of the previous section the effective vector nature of the new interaction has been established. Therefore measurement of $R$ in the xenon detector fixes $\tilde{\xi}_{V}=\tilde{\xi} \mid \mathrm{Xe}$. This information can be used to fix the overall factor $F_{V} h_{V}^{p}$ to $F_{V} h_{V}^{p}=\left.\tilde{\xi}\right|_{\mathrm{Xe}} /\left(Z_{\mathrm{Xe}}+N_{\mathrm{Xe}} r_{V}\right)$. With that information as an input one can then calculate the number of events in a second detector (D2) as a function of $r_{V}$. The value of $r_{V}$ for which $N_{\text {theory }}^{\text {D2 }}=N_{\text {Exp }}^{\mathrm{D} 2}$ will tell whether the new interaction is isospin conserving or violating: $r_{V}=1$ will establish isospin conservation, while any other case will prove otherwise. Note that the protophobic and neutrophic scenarios will be favored by $r_{V} \ll 1$ and $r_{V} \gg 1$, respectively.

To show the performance of different detectors we take the parameter space point for which $R=1.08$, obtained for $\left.\tilde{\xi}_{V}\right|_{\mathrm{Xe}}=74.77$ (see left graph in figure 4 ). With the overall factor in (4.4) fixed according to this number, we then calculate the expected number of events in argon and silicon detectors as a function of $r_{V}$. The result is displayed in the left graph in figure 5, which shows the number of events in each detector as a function of $r_{V}$. Although done for a particular point in parameter space, this result allows to capture the general picture. Light isotopes have a stronger $r_{V}$ dependence, so are better suited to determine the isospin character of the new interaction. For silicon and for $r_{V}$ varying as shown in figure 5 (left graph), $N_{\text {events }}$ decreases about $63 \%$ while for argon about 39\% (we checked for germanium as well and in that case the variation is smaller). Note that this conclusion is inline with analysis done in the context of isospin violating DM [79]. The number of events increases in the neutrophobic case and decreases in protophobic scenarios. Such behavior can therefore be used to identify the scenario to 
which the new physics belongs. The results displayed in the left graph in figure 5 are derived by assuming a $0.1 \mathrm{keV}$ threshold. If one takes instead more realistic (experimentally) values, the number of events can drastically decrease as shown in the right graph in figure 5 . In such a case larger exposures will be required so to determine the isospin nature of the new interaction, something feasibale at e.g. the Argo detector of the Global Argon Dark Matter Collaboration [78].

A second measurement then fixes $r_{V}$ - of course within experimental uncertainties - and that information can be used to partially reconstruct the parameter space $h_{V}^{u, d}$ of the model responsible for the signal. If it turns out that the model is protophobic or neutrophobic partial reconstruction can be done right away with the aid of eqs. (4.2). It is worth pointing out that the procedure outlined here applies in the same manner to the effective scalar case. It applies as well in light mediator scenarios but in those cases $r_{X}$ is determined within a range, as we now explain. Measurement in the xenon detector provides $R=\left.R\right|_{\text {Xe }}$ and fixes the nature of the new interaction to, say, light scalar mediator. This value for $R$ is obtained not for a single value of $F_{S} C_{S}^{N}$, but within the interval $\mathcal{I}=\left[\left.F_{S} C_{S}^{N}\right|_{\min },\left.F_{S} C_{S}^{N}\right|_{\max }\right]$ (see left graph in figure 3). For the overall factor in eq. (4.5) this translates into $F_{S} h_{S}^{p}=\mathcal{I} /\left(Z_{\mathrm{Xe}}+N_{\mathrm{Xe}} r_{S}\right)$, thus leading to an spread in the calculation of $N_{\text {theory }}^{\mathrm{D} 2}$ in terms of $r_{s}$.

\subsection{The case of degeneracies}

We now turn to the discussion of parameter space degeneracies. For that aim we will assume that degeneracy happens in a xenon detector and will determine which among the silicon, argon and germanium detectors performs better at breaking the degeneracy. For such choice and according to eqs. (4.2) and (4.3) the proton and scalar couplings can be entirely expressed in terms of $h_{X}^{d}$

$$
\begin{aligned}
& \text { Vector : } \quad h_{V}^{p}=-1.252 h_{V}^{d}, \quad h_{V}^{n}=0.874 h_{V}^{d} \text {, } \\
& \text { Scalar : } \quad h_{S}^{p}=-0.964 h_{S}^{d}, \quad h_{S}^{n}=0.672 h_{S}^{d} \text {. }
\end{aligned}
$$

With these results one can evaluate the nuclear coupling in silicon, argon and germanium in terms only of $h_{X}^{d}$

$$
\begin{array}{lll}
\left.C_{V}^{N}\right|_{\mathrm{Si}}=-5.2 h_{V}^{d}, & \left.C_{V}^{N}\right|_{\mathrm{Ar}}=-3.3 h_{V}^{d}, & \left.C_{V}^{N}\right|_{\mathrm{Ge}}=-4.5 h_{V}^{d}, \\
\left.C_{S}^{N}\right|_{\mathrm{Si}}=-4.0 h_{S}^{d}, & \left.C_{S}^{N}\right|_{\mathrm{Ar}}=-2.5 h_{S}^{d}, & \left.C_{S}^{N}\right|_{\mathrm{Ge}}=-3.5 h_{S}^{d} .
\end{array}
$$

For the calculation of the number of events in the different detectors and for the effective case we then vary $F_{V} h_{V}^{d}$ within the interval $[-1,0]$ to assure $\xi_{V}>0$, as required by (2.16). $F_{S} h_{S}^{d}$ we instead vary within $[-1,1]$. For light mediators we vary $h_{X}^{d}$ within $\left[10^{-9}, 10^{-7}\right]$, to - again - guarantee constraints from COHERENT are satisfied. We calculate as well the SM expectation for each isotope:

$$
\left.N_{\text {Event }}^{\mathrm{SM}}\right|_{\mathrm{Si}}=136.5,\left.\quad N_{\text {Event }}^{\mathrm{SM}}\right|_{\mathrm{Ar}}=234.5,\left.\quad N_{\text {Event }}^{\mathrm{SM}}\right|_{\mathrm{Ge}}=417.8 .
$$

The results are shown in figure 6. From the graphs on top, which correspond to the effective vector (left) and scalar (right) cases, one can see that silicon performs better than argon and 

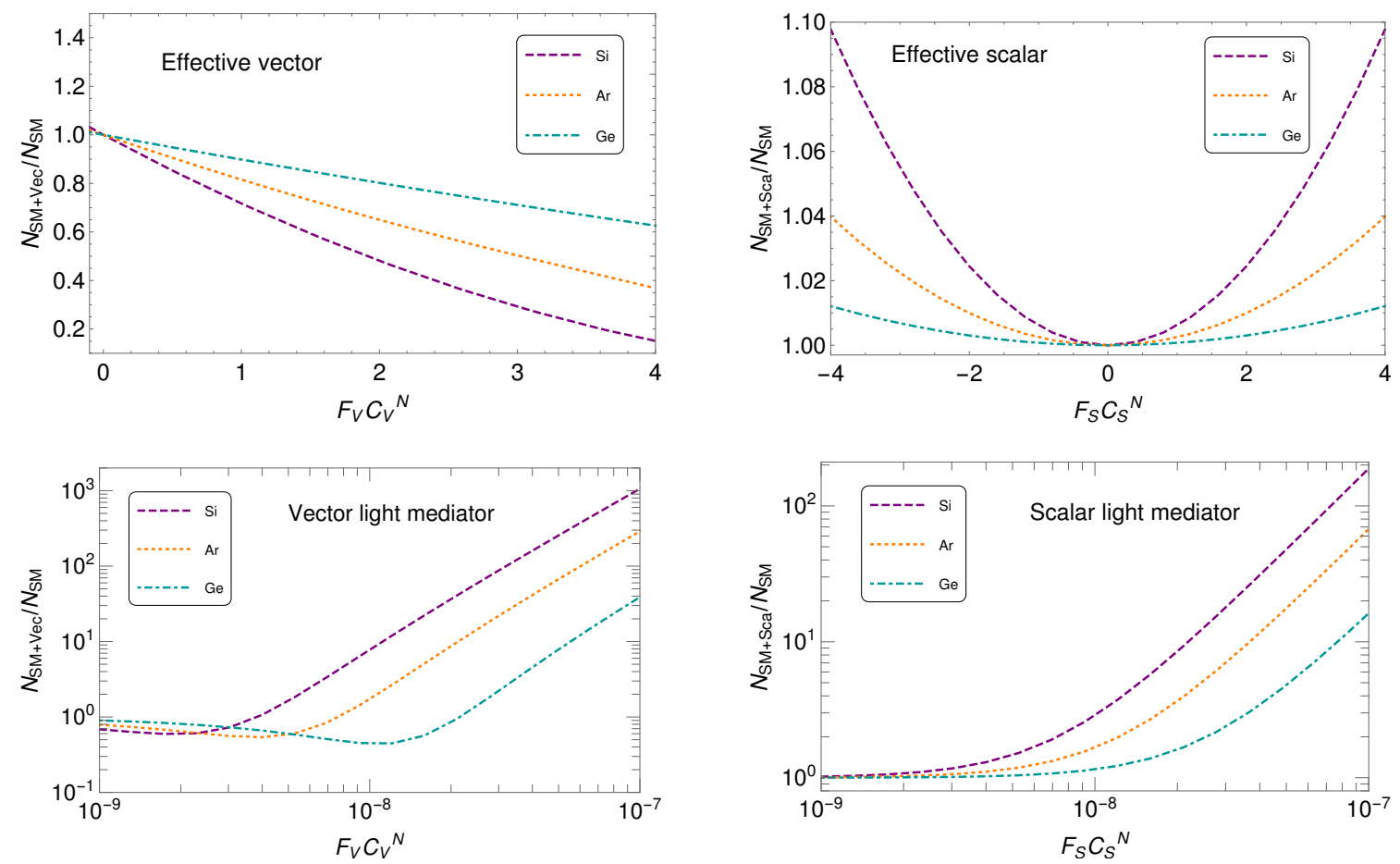

Figure 6. Top-left graph: number of events normalized to the SM expectation in silicon, argon and germanium detectors assuming parameter space degeneracy in a xenon detector $\left.C_{X}^{N}\right|_{\mathrm{Xe}}=0$. The result was obtained assuming $E_{r}^{\text {th }}=0.1 \mathrm{keV}$ and an effective vector interaction. Top-right graph Same as in the top-left graph but for effective scalar interactions. Both results demonstrate that the silicon detector performs better for degeneracy removal and allow to distinguish in almost all parameter space vector from scalar signals. Bottom-left graph: same as in the top-left graph but for vector light mediators. As in the effective case — in general — silicon performs better. However in contrast to the effective case the signal is more degraded (below the SM) in germanium, so whether silicon or germanium are more suited for breaking xenon degeneracies depends on where the values of the parameters fall in. Bottom-right graph: same as in the top-left graph but for scalar light mediators. For light mediators scenarios the calculation has been done fixing $m_{X}=1 \mathrm{MeV}$, value for which effects are maximized (within the mass range we are considering).

germanium. The vector interaction depletes $N_{\text {Event }}$ below the SM expectation. For silicon the depletion amounts to about $0.1 \times N_{\text {Event }}^{\mathrm{SM}} \mid \mathrm{Si}$. For scalar interactions deviations from the SM prediction are somehow more modest, in silicon they amount at most to $10 \%$. Thus, breaking scalar degeneracies in xenon will require a silicon detector with a high event rate resolution. All in all, among the isotopes we are considering, study of degeneracies in xenon generated by effective interactions should be — ideally — done combining measurements in xenon and silicon detectors.

From the graphs on top one can see as well that identification of vector and scalar interactions can be done in all the parameter space we are considering just by measurements of $R$. Depletions below the SM expectation will establish vector interactions as responsible for the signal. Enhancements, instead, will favor scalar interactions. Measurements in a silicon detector will therefore not only remove the parameter space degeneracy but will also establish the nature of the new physics. 
In the case of light mediators silicon performs - in general - better than argon and germanium. However, as shown in the bottom-left graph, for vectors germanium is more sensitive to depletions than what silicon and argon are. The question of xenon degeneracy breaking thus depends to a large extent on the window where the parameters happen to fall in. For germanium, in the range $F_{V} C_{V}^{N} \gtrsim[0.5,3.0] \times 10^{-8}$ the signal is depleted below the $\mathrm{SM}$ expectation with values as small as $\left.0.4 N_{\mathrm{Event}}^{\mathrm{SM}}\right|_{\mathrm{SM}} \simeq 167.1$. For values $F_{V} C_{V}^{N} \gtrsim 3.0 \times 10^{-8}$ the signal gets enhanced, with those enhancements leading to signals that can exceed the SM prediction by three orders of magnitude in silicon. For light scalars, values of the couplings at $10^{-8}$ lead to enhancements of order 3 , as shown in the bottom-right graph. Above those values the signal can exceed the SM expectation by more than a factor $10^{2}$, in silicon as well.

After measurements in silicon are carried out they can be used as well to distinguish vector from scalar interactions, at least in certain regions of parameter space. Comparing the bottom-left and bottom-right graphs in figure 6 one can see that vectors produce enhancements that scalars cannot reach. Thus measuring $R$ above $\sim 200$ will provide an experimental prove that a new light vector boson is at work. As we discussed in section 3, observation of depletions below the SM prediction will favor vector interactions over scalar, and that applies in this case too. In the region $R \cong[1.1,200]$, one finds the same situation discussed in section 3: a counting experiment alone cannot distinguish vector and scalar signals. In that case information of the recoil spectrum is required to gain information on the nature of the new interaction.

We close this section by stressing that measurements of $\mathrm{CE} \nu \mathrm{NS}$ in xenon detectors matching the SM prediction not necessarily rule out the presence of new physics. In that case our results encourage measurements in any of the target materials we have considered, but ideally in silicon.

\section{CE $\nu$ NS and new interactions in XENON1T and LZ}

We now quantify the modifications that the interactions in (2.1) and (2.2) introduce on the $\mathrm{CE} \nu \mathrm{NS}$ event rate spectrum for on-going and future Xenon-based experiments $[3,80]$. Since for both cases we use a simplified acceptance function $\mathcal{A}\left(E_{r} / \mathrm{keV}\right)=H\left(1-E_{r} / \mathrm{keV}\right)$, the differences between the spectra arises only from exposure. For XENON1T we take $\mathcal{E}_{\mathrm{Xe1T}}=2$ ton-year while for LZ $\mathcal{E}_{\mathrm{LZ}}=10$ ton-year. We therefore present only results for XENON1T, results for LZ are obtained by a factor 5 rescaling.

With this detector specifications we then calculate the event rate spectrum first for light mediator scenarios and then for effective interactions. To do so we chose parameters that maximize enhancements/depletions above/below the SM expectaction (see sections 3.1 and 3.2). The results are displayed in figure 7. For light mediators (left graph) one can see that in both cases the number of $\mathrm{CE} \nu \mathrm{NS}$ events can readily exceed by far the SM expectation, provided thresholds are pushed below $2 \mathrm{keV}$ or so. For the parameter combinations that maximizes the event rate we find $N_{\text {Events }}^{\mathrm{Vec}} \simeq 1643$ and $N_{\mathrm{Events}}^{\mathrm{Sca}} \simeq 4340$, to be compared with the $\mathrm{SM}$ expectation $N_{\mathrm{Events}}^{\mathrm{SM}} \simeq 189$. It is worth stressing that lower thresholds, e.g $0.1 \mathrm{keV}$ as has been used for the analyses in section 3 , tend to diminsh 

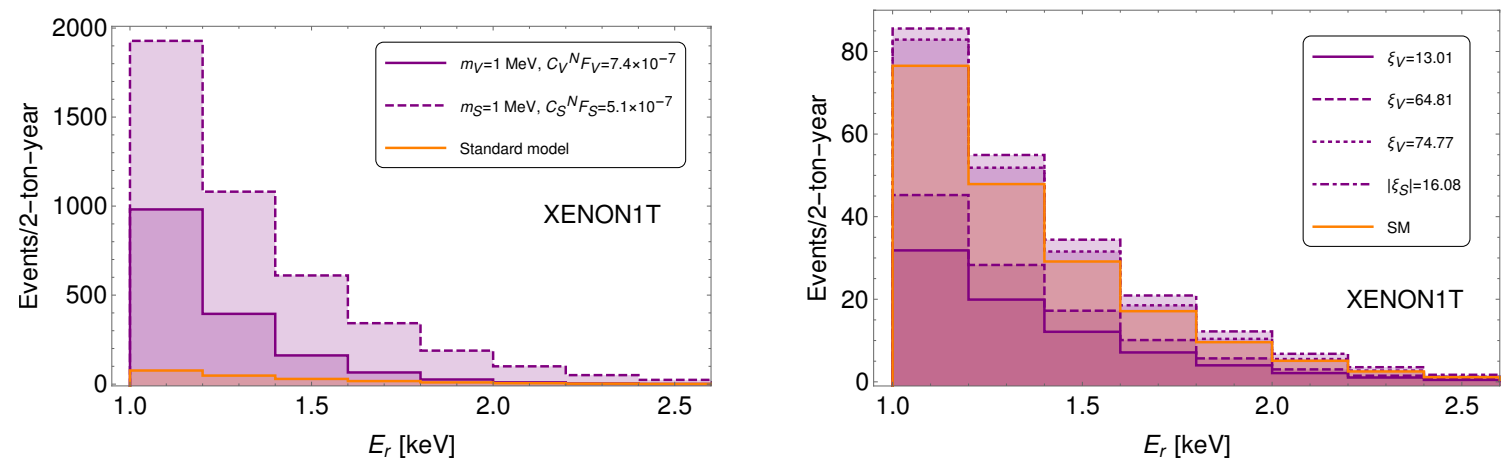

Figure 7. Left graph: expected event rate spectrum for vector and scalar light mediators at XENON1T assuming a 2 ton-year exposure. Parameters are chosen to maximize the new physics event rate. Any other choice will therefore lead to events below these values. Right graph: expected event rate spectrum for vector and scalar effective interactions. Results for LZ (10 ton-year) can be directly derived by rescaling these event rate spectrum by a factor 5 . For comparison the SM expectation is also shown (orange histogram). These results demonstrate that low recoil threshold measurements will provide sufficient statistics with which precise studies of new interactions can be done.

the scalar-induced event rate compared to the vector. This can be easily understood from the vector and scalar differential cross sections in eqs. (2.3) and (2.4). The scalar being proportional to $E_{r}$ gets depleted at low thresholds, something that does not happen with the vector. Thus, measuments at, say, $0.1 \mathrm{keV}$ somehow favor detection of vector interaction signals, on the contrary at $1 \mathrm{keV}$ detection of scalar signals is favored instead. The same conclusion is found in the effective limits as well.

For effective interactions (right graph) results are rather different as a consequence of the possible (maximum/minimum) values the effective vector and scalar couplings can have, resulting in deviations from the SM expectation not as pronounced as they are in the light mediator case. Vector interactions can yield up to $N_{\text {Events }}^{\mathrm{Vec}} \simeq 205$, with that number diminished down to $N_{\text {Events }}^{\mathrm{Vec}} \simeq 112$ or $N_{\text {Events }}^{\mathrm{Vec}} \simeq 79$ depending on parameter choice. Note that depletions below the SM expectation are found as well in the vector light mediator case (see left graph in figure 3), so observation of event rates below those of the SM will be a sufficient criteria to establish the vector nature of the new interaction. For the scalar coupling case we find $N_{\text {Events }}^{\text {Sca }} \simeq 221$, as expected always exceeding the SM prediction. In summary, in both cases (vector and scalar) the number of events exceeds (or goes below) the SM expectation with an statistics that might be sufficient to identify the new interaction as well as to eventually reconstruct its "morphology".

\section{Conclusions}

In this paper we studied the sensitivity of multi-ton DM experiments to distinguish vector and scalar new physics signals, through measurements of $\mathrm{CE} \nu \mathrm{NS}$ processes induced by ${ }^{8} \mathrm{~B}$ neutrinos. We addressed the question of whether given a signal not matching the SM expectation those measurements can identify the nature of the new interaction (assuming the $\mathrm{CE} \nu \mathrm{NS}$ signal can be differentiated from a DM signal). We considered the case of light 
mediators as well as the effective case. The former taken deep inside the light mediator window $[1,10] \mathrm{MeV}$, while the latter valid for $m_{X} \gtrsim 10^{3} \mathrm{MeV}$. We used the recent COHERENT timing and energy data and neutrino mass scales to apply constraints on both scalar and vector interactions for light and heavy mediators before we investigate them at the DM detectors. Using a xenon-based DM detector we identified cases where measurements of the event spectrum alone suffices to establish whether the signal is due to vector or scalar couplings. For light mediators, measurements yielding $R=N_{\mathrm{SM}+\mathrm{Vec}} / N_{\mathrm{SM}} \gtrsim 84$ or $R \lesssim 1$ will demonstrate the presence of a new light gauge boson, while discarding a dominant light scalar contribution. In the effective case, instead, measurements resulting in $R \gtrsim 1.05$ or $R \lesssim 1$ will demonstrate that a "heavy" vector mediator is at work.

We identified as well a region of degeneracy where a deviation above the SM prediction can be accounted for by either the vector or the scalar interaction. In the light mediator case we found that for measurements resulting in $R \cong(1,84]$, disentanglement of the vector and scalar contributions cannot be done only by measurements of the event spectrum. In the effective case we found that such region exist as well for $R \cong(1,1.05]$. We showed that such vector-scalar signal degeneracy can be broken by combined measurements of the event and recoil spectra. In the light mediator case identification of vector interactions is possible because there is always a dip in their recoil spectrum for recoil energies between $0.1-3.5 \mathrm{keV}$, whereas for scalars this is never the case. In the effective case, scalar interactions lead to recoil spectra that fall more steeply than vector recoil spectra do. Combined measurements of number of events and recoil spectra can then be used to identify the origin of the new physics signal.

We considered as well the capability of multi-ton DM detectors to measure the couplings of the new mediator to protons and neutrons, or in other words to determine whether the new physics is or not isospin conserving. For that aim we considered xenon, silicon, argon and germanium detectors and focused on three extreme scenarios: protophobia, neutrophobia and degeneracies. The latter defined as a scenario in which the new physics exactly cancels in a particular nuclide. We showed that in the effective case two independent measurements of the event spectrum are sufficient to pin down the value of the neutron-to-proton couplings ratio, and so to establish whether the new physics conserves or breaks isospin. We demonstrated that to establish the isospin nature of the new interaction, given a first measurement in xenon, silicon is the most suited nuclide among those we considered. We stressed that in contrast to measurements of these type for DM, in this case statistics is - in principle - not an issue and so implementation of such detector complementarity should be feasible.

We studied the case of parameter space degeneracies assuming the new physics signal exactly vanishes in xenon. We showed that degeneracy breaking can be done in any of the detectors considered, but silicon performs way better in both cases, light and effective limits. The exception being only a particular region in parameter space for light vector mediators, where depletions in germanium are more pronounced than in argon and silicon. We pointed out that if measurements in xenon match the SM expectation, efforts towards measuring $\mathrm{CE} \nu \mathrm{NS}$ in other ton-size detectors using silicon should be carried out to test whether new physics is hidden in measurements involving xenon. 
Finally, we found that the new vector and scalar interactions can be investigated with $\sim 210-4000$ events (for parameters that maximize the event rate) with a 2 tonyear experiment (e.g. XENON1T), or five times those values for a 10 ton-year exposure (e.g. XENONnT and LZ), if the nuclear recoil energy threshold is reduced down to $1 \mathrm{keV}$. Depletions below the SM expectation can be expected too. With still sufficient statistics, $\sim 80-110$, they will demonstrate that the origin of the new signal arises from a new vector force. Note that the current thresholds $\sim 5 \mathrm{keV}$ (XENON1T) and $4.4 \mathrm{keV}$ (LZ) would not apply any constraints on scalar and vector interactions in the ${ }^{8} \mathrm{~B}$ solar neutrinos. We also stress that the results presented here apply as well to other $\mathrm{CE} \nu \mathrm{NS}-$ related experiments such as CONNIE [11], CONUS [12] and $\nu$-cleus [13]. Interpretation of data from these experiments in terms of new physics should take into account the results we have presented in this paper.

\section{Acknowledgments}

DAS is supported by the grant "Unraveling new physics in the high-intensity and highenergy frontiers", Fondecyt No. 1171136. He would like to thank Nicolas Rojas for useful conversations. The work of BD, SL and LS are supported in part by the DOE Grant No. DE-SC0010813. DAS would like to thank the University of Texas A\&M Mitchell Institute for Fundamental Physics for its hospitality.

Open Access. This article is distributed under the terms of the Creative Commons Attribution License (CC-BY 4.0), which permits any use, distribution and reproduction in any medium, provided the original author(s) and source are credited.

\section{References}

[1] J. Billard, L. Strigari and E. Figueroa-Feliciano, Implication of neutrino backgrounds on the reach of next generation dark matter direct detection experiments, Phys. Rev. D 89 (2014) 023524 [arXiv: 1307.5458] [INSPIRE].

[2] XENON collaboration, Physics reach of the XENON1T dark matter experiment, JCAP 04 (2016) 027 [arXiv:1512.07501] [INSPIRE].

[3] LUX-ZEPLIN collaboration, Projected WIMP Sensitivity of the LUX-ZEPLIN (LZ) Dark Matter Experiment, arXiv:1802.06039 [INSPIRE].

[4] DARWIN collaboration, DARWIN: towards the ultimate dark matter detector, JCAP 11 (2016) 017 [arXiv: 1606.07001] [INSPIRE].

[5] P. Grothaus, M. Fairbairn and J. Monroe, Directional Dark Matter Detection Beyond the Neutrino Bound, Phys. Rev. D 90 (2014) 055018 [arXiv:1406.5047] [InSPIRE].

[6] C.A.J. O’Hare, A.M. Green, J. Billard, E. Figueroa-Feliciano and L.E. Strigari, Readout strategies for directional dark matter detection beyond the neutrino background, Phys. Rev. D 92 (2015) 063518 [arXiv: 1505.08061] [INSPIRE].

[7] J.H. Davis, Dark Matter vs. Neutrinos: The effect of astrophysical uncertainties and timing information on the neutrino floor, JCAP 03 (2015) 012 [arXiv:1412.1475] [INSPIRE]. 
[8] F. Ruppin, J. Billard, E. Figueroa-Feliciano and L. Strigari, Complementarity of dark matter detectors in light of the neutrino background, Phys. Rev. D 90 (2014) 083510 [arXiv: 1408.3581] [INSPIRE].

[9] COHERENT collaboration, Observation of Coherent Elastic Neutrino-Nucleus Scattering, Science 357 (2017) 1123 [arXiv: 1708.01294] [INSPIRE].

[10] COHERENT collaboration, COHERENT Collaboration data release from the first observation of coherent elastic neutrino-nucleus scattering, arXiv:1804.09459 [INSPIRE].

[11] CONNIE collaboration, Exploring low-energy neutrino physics with the Coherent Neutrino Nucleus Interaction Experiment, Phys. Rev. D 100 (2019) 092005 [arXiv:1906. 02200] [INSPIRE].

[12] C. Buck et al., The CONUS Experiment, https://indico.cern.ch/event/606690/contributions/ 2591545/attachments/1499330/2336272/Taup2017_CONUS_talk_JHakenmueller.pdf.

[13] R. Strauss et al., The $\nu$-cleus experiment: A gram-scale fiducial-volume cryogenic detector for the first detection of coherent neutrino-nucleus scattering, Eur. Phys. J. C 77 (2017) 506 [arXiv: 1704.04320] [INSPIRE].

[14] B. Dutta, S. Liao, L.E. Strigari and J.W. Walker, Non-standard interactions of solar neutrinos in dark matter experiments, Phys. Lett. B 773 (2017) 242 [arXiv:1705.00661] [INSPIRE].

[15] D. Aristizabal Sierra, N. Rojas and M.H.G. Tytgat, Neutrino non-standard interactions and dark matter searches with multi-ton scale detectors, JHEP 03 (2018) 197 [arXiv: 1712.09667] [INSPIRE].

[16] M.C. Gonzalez-Garcia, M. Maltoni, Y.F. Perez-Gonzalez and R. Zukanovich Funchal, Neutrino Discovery Limit of Dark Matter Direct Detection Experiments in the Presence of Non-Standard Interactions, JHEP 07 (2018) 019 [arXiv: 1803.03650] [INSPIRE].

[17] D.G. Cerdeño, M. Fairbairn, T. Jubb, P.A.N. Machado, A.C. Vincent and C. Boehm, Physics from solar neutrinos in dark matter direct detection experiments, JHEP 05 (2016) 118 [Erratum ibid. 09 (2016) 048] [arXiv: 1604.01025] [INSPIRE].

[18] D. Aristizabal Sierra, V. De Romeri and N. Rojas, COHERENT analysis of neutrino generalized interactions, Phys. Rev. D 98 (2018) 075018 [arXiv:1806.07424] [INSPIRE].

[19] J.L. Newstead, L.E. Strigari and R.F. Lang, Detecting CNO solar neutrinos in next-generation xenon dark matter experiments, Phys. Rev. D 99 (2019) 043006 [arXiv: 1807.07169] [INSPIRE].

[20] R.F. Lang, C. McCabe, S. Reichard, M. Selvi and I. Tamborra, Supernova neutrino physics with xenon dark matter detectors: A timely perspective, Phys. Rev. D 94 (2016) 103009 [arXiv: 1606. 09243] [INSPIRE].

[21] M. Lindner, W. Rodejohann and X.-J. Xu, Coherent Neutrino-Nucleus Scattering and new Neutrino Interactions, JHEP 03 (2017) 097 [arXiv: 1612.04150] [INSPIRE].

[22] J.B. Dent, B. Dutta, J.L. Newstead and L.E. Strigari, Dark matter, light mediators and the neutrino floor, Phys. Rev. D 95 (2017) 051701 [arXiv: 1607.01468] [INSPIRE].

[23] Y. Farzan, M. Lindner, W. Rodejohann and X.-J. Xu, Probing neutrino coupling to a light scalar with coherent neutrino scattering, JHEP 05 (2018) 066 [arXiv:1802.05171] [INSPIRE].

[24] J. Liao and D. Marfatia, COHERENT constraints on nonstandard neutrino interactions, Phys. Lett. B 775 (2017) 54 [arXiv:1708.04255] [INSPIRE]. 
[25] V. Brdar, W. Rodejohann and X.-J. Xu, Producing a new Fermion in Coherent Elastic Neutrino-Nucleus Scattering: from Neutrino Mass to Dark Matter, JHEP 12 (2018) 024 [arXiv: 1810.03626] [INSPIRE].

[26] D. Aristizabal Sierra, V. De Romeri and N. Rojas, CP violating effects in coherent elastic neutrino-nucleus scattering processes, JHEP 09 (2019) 069 [arXiv:1906.01156] [INSPIRE].

[27] G. Arcadi, M. Lindner, J. Martins and F.S. Queiroz, New Physics Probes: Atomic Parity Violation, Polarized Electron Scattering and Neutrino-Nucleus Coherent Scattering, arXiv: 1906.04755 [INSPIRE].

[28] D.K. Papoulias and T.S. Kosmas, COHERENT constraints to conventional and exotic neutrino physics, Phys. Rev. D 97 (2018) 033003 [arXiv:1711.09773] [InSPIRE].

[29] D.K. Papoulias, COHERENT constraints after the Chicago-3 quenching factor measurement, arXiv:1907.11644 [INSPIRE].

[30] O.G. Miranda, D.K. Papoulias, M. Tórtola and J.W.F. Valle, Probing neutrino transition magnetic moments with coherent elastic neutrino-nucleus scattering, JHEP 07 (2019) 103 [arXiv: 1905.03750] [INSPIRE].

[31] O.G. Miranda, G. Sanchez Garcia and O. Sanders, Coherent elastic neutrino-nucleus scattering as a precision test for the Standard Model and beyond: the COHERENT proposal case, Adv. High Energy Phys. 2019 (2019) 3902819 [arXiv: 1902.09036] [INSPIRE].

[32] X.-J. Xu, Tensor and scalar interactions of neutrinos may lead to observable neutrino magnetic moments, Phys. Rev. D 99 (2019) 075003 [arXiv:1901.00482] [INSPIRE].

[33] I. Bischer and W. Rodejohann, General Neutrino Interactions at the DUNE Near Detector, Phys. Rev. D 99 (2019) 036006 [arXiv: 1810.02220] [INSPIRE].

[34] I. Bischer and W. Rodejohann, General neutrino interactions from an effective field theory perspective, Nucl. Phys. B 947 (2019) 114746 [arXiv: 1905. 08699] [INSPIRE].

[35] A.N. Khan, W. Rodejohann and X.-J. Xu, Borexino and General Neutrino Interactions, arXiv: 1906.12102 [INSPIRE].

[36] D. Aristizabal Sierra, J. Liao and D. Marfatia, Impact of form factor uncertainties on interpretations of coherent elastic neutrino-nucleus scattering data, JHEP 06 (2019) 141 [arXiv: 1902.07398] [INSPIRE].

[37] R.H. Helm, Inelastic and Elastic Scattering of 187-Mev Electrons from Selected Even-Even Nuclei, Phys. Rev. 104 (1956) 1466 [InSPIRE].

[38] I. Angeli and K.P. Marinova, Table of experimental nuclear ground state charge radii: An update, Atom. Data Nucl. Data Tabl. 99 (2013) 69.

[39] Particle Data Group collaboration, Review of Particle Physics, Chin. Phys. C 40 (2016) 100001 [INSPIRE].

[40] A. Crivellin, M. Hoferichter and M. Procura, Accurate evaluation of hadronic uncertainties in spin-independent WIMP-nucleon scattering: Disentangling two- and three-flavor effects, Phys. Rev. D 89 (2014) 054021 [arXiv:1312.4951] [INSPIRE].

[41] M. Hoferichter, J. Ruiz de Elvira, B. Kubis and U.-G. Meissner, High-Precision Determination of the Pion-Nucleon $\sigma$ Term from Roy-Steiner Equations, Phys. Rev. Lett. 115 (2015) 092301 [arXiv:1506.04142] [INSPIRE].

[42] J. Ellis, N. Nagata and K.A. Olive, Uncertainties in WIMP Dark Matter Scattering Revisited, Eur. Phys. J. C 78 (2018) 569 [arXiv:1805.09795] [INSPIRE]. 
[43] M. Bauer, P. Foldenauer and J. Jaeckel, Hunting All the Hidden Photons, JHEP 07 (2018) 094 [arXiv: 1803. 05466] [INSPIRE].

[44] LHCb collaboration, Search for Dark Photons Produced in 13 TeV pp Collisions, Phys. Rev. Lett. 120 (2018) 061801 [arXiv:1710.02867] [INSPIRE].

[45] KLOE-2 collaboration, Limit on the production of a new vector boson in $\mathrm{e}^{+} \mathrm{e}^{-} \rightarrow \mathrm{U} \gamma$, $\mathrm{U} \rightarrow \pi^{+} \pi^{-}$with the KLOE experiment, Phys. Lett. B 757 (2016) 356 [arXiv:1603.06086] [INSPIRE].

[46] BABAR collaboration, Search for a Dark Photon in $e^{+} e^{-}$Collisions at BaBar, Phys. Rev. Lett. 113 (2014) 201801 [arXiv: 1406 . 2980] [INSPIRE].

[47] G. Inguglia, Belle II studies of missing energy decays and searches for dark photon production, PoS (DIS2016) 263 (2016) [arXiv: 1607.02089] [INSPIRE].

[48] D. Curtin, R. Essig, S. Gori and J. Shelton, Illuminating Dark Photons with High-Energy Colliders, JHEP 02 (2015) 157 [arXiv:1412.0018] [INSPIRE].

[49] CHARM collaboration, Experimental Verification of the Universality of $\nu_{e}$ and $\nu_{\mu}$ Coupling to the Neutral Weak Current, Phys. Lett. B 180 (1986) 303 [INSPIRE].

[50] CCFR collaboration, Neutrino tridents and W Z interference, Phys. Rev. Lett. 66 (1991) 3117 [INSPIRE].

[51] NuTeV collaboration, A Precise Determination of Electroweak Parameters in Neutrino Nucleon Scattering, Phys. Rev. Lett. 88 (2002) 091802 [Erratum ibid. 90 (2003) 239902] [hep-ex/0110059] [INSPIRE].

[52] Y. Kaneta and T. Shimomura, On the possibility of a search for the $L_{\mu}-L_{\tau}$ gauge boson at Belle-II and neutrino beam experiments, PTEP 2017 (2017) 053B04 [arXiv:1701.00156] [INSPIRE].

[53] S. Bilmis, I. Turan, T.M. Aliev, M. Deniz, L. Singh and H.T. Wong, Constraints on Dark Photon from Neutrino-Electron Scattering Experiments, Phys. Rev. D 92 (2015) 033009 [arXiv: 1502.07763] [INSPIRE].

[54] M. Lindner, F.S. Queiroz, W. Rodejohann and X.-J. Xu, Neutrino-electron scattering: general constraints on $Z^{\prime}$ and dark photon models, JHEP 05 (2018) 098 [arXiv: 1803.00060] [INSPIRE].

[55] M.B. Wise and Y. Zhang, Effective Theory and Simple Completions for Neutrino Interactions, Phys. Rev. D 90 (2014) 053005 [arXiv: 1404.4663] [INSPIRE].

[56] MiniBoonE collaboration, First Measurement of the Muon Neutrino Charged Current Quasielastic Double Differential Cross Section, Phys. Rev. D 81 (2010) 092005 [arXiv: 1002.2680] [INSPIRE].

[57] MiniBooNE collaboration, First measurement of the muon antineutrino double-differential charged-current quasielastic cross section, Phys. Rev. D 88 (2013) 032001 [arXiv: 1301.7067] [INSPIRE].

[58] MiniBooNE collaboration, Measurement of the Neutrino Neutral-Current Elastic Differential Cross Section on Mineral Oil at $E_{\nu} \sim 1$ GeV, Phys. Rev. D 82 (2010) 092005 [arXiv: 1007.4730] [INSPIRE].

[59] NOMAD collaboration, A Measurement of Coherent Neutral Pion Production in Neutrino Neutral Current Interactions in NOMAD, Phys. Lett. B 682 (2009) 177 [arXiv:0910.0062] [INSPIRE]. 
[60] MINERvA collaboration, Measurement of Muon Neutrino Quasielastic Scattering on a Hydrocarbon Target at $E_{\nu} \sim 3.5$ GeV, Phys. Rev. Lett. 111 (2013) 022502

[arXiv: 1305.2243] [INSPIRE].

[61] P. Coloma, P.B. Denton, M.C. Gonzalez-Garcia, M. Maltoni and T. Schwetz, Curtailing the Dark Side in Non-Standard Neutrino Interactions, JHEP 04 (2017) 116 [arXiv:1701.04828] [INSPIRE].

[62] J.I. Collar, A.R.L. Kavner and C.M. Lewis, Response of CsI[Na] to Nuclear Recoils: Impact on Coherent Elastic Neutrino-Nucleus Scattering (CEעNS), Phys. Rev. D 100 (2019) 033003 [arXiv: 1907.04828] [INSPIRE].

[63] A.N. Khan and W. Rodejohann, New Physics from COHERENT Data with Improved Quenching Factors, arXiv: 1907.12444 [INSPIRE].

[64] B. Dutta, S. Liao, S. Sinha and L.E. Strigari, Searching for Beyond the Standard Model Physics with COHERENT Energy and Timing Data, Phys. Rev. Lett. 123 (2019) 061801 [arXiv: 1903.10666] [INSPIRE].

[65] C. Giunti, General COHERENT Constraints on Neutrino Non-Standard Interactions, arXiv: 1909.00466 [INSPIRE].

[66] Y. Nambu and G. Jona-Lasinio, Dynamical Model of Elementary Particles Based on an Analogy with Superconductivity. 1., Phys. Rev. 122 (1961) 345 [InSPIRE].

[67] A.M. Bernstein and B.R. Holstein, Neutral Pion Lifetime Measurements and the QCD Chiral Anomaly, Rev. Mod. Phys. 85 (2013) 49 [arXiv:1112.4809] [InSPIRE].

[68] Planck collaboration, Planck 2018 results. VI. Cosmological parameters, arXiv:1807.06209 [inSPIRE].

[69] J.A. Grifols and E. Masso, Constraints on Finite Range Baryonic and Leptonic Forces From Stellar Evolution, Phys. Lett. B 173 (1986) 237 [INSPIRE].

[70] J.A. Grifols, E. Masso and S. Peris, Energy Loss From the Sun and RED Giants: Bounds on Short Range Baryonic and Leptonic Forces, Mod. Phys. Lett. A 4 (1989) 311 [InSPIRE].

[71] J.H. Chang, R. Essig and S.D. McDermott, Revisiting Supernova 1987A Constraints on Dark Photons, JHEP 01 (2017) 107 [arXiv:1611.03864] [INSPIRE].

[72] B. Müller, The Status of Multi-Dimensional Core-Collapse Supernova Models, Publ. Astron. Soc. Austral. 33 (2016) e048 [arXiv: 1608.03274] [InSPIRE].

[73] E. Hardy and R. Lasenby, Stellar cooling bounds on new light particles: plasma mixing effects, JHEP 02 (2017) 033 [arXiv: 1611.05852] [INSPIRE].

[74] A.E. Nelson and J. Walsh, Short Baseline Neutrino Oscillations and a New Light Gauge Boson, Phys. Rev. D 77 (2008) 033001 [arXiv:0711.1363] [inSPIRE].

[75] A.E. Nelson and J. Walsh, Chameleon vector bosons, Phys. Rev. D 77 (2008) 095006 [arXiv: 0802.0762] [INSPIRE].

[76] J.N. Bahcall, A.M. Serenelli and S. Basu, New solar opacities, abundances, helioseismology and neutrino fluxes, Astrophys. J. 621 (2005) L85 [astro-ph/0412440] [INSPIRE].

[77] J. Heeck, M. Lindner, W. Rodejohann and S. Vogl, Non-Standard Neutrino Interactions and Neutral Gauge Bosons, SciPost Phys. 6 (2019) 038 [arXiv:1812.04067] [INSPIRE].

[78] C.E. Aalseth et al., DarkSide-20k: A 20 tonne two-phase LAr TPC for direct dark matter detection at LNGS, Eur. Phys. J. Plus 133 (2018) 131 [arXiv:1707.08145] [INSPIRE]. 
[79] C. Kelso, J. Kumar, D. Marfatia and P. Sandick, Directly detecting Isospin-Violating Dark Matter, Phys. Rev. D 97 (2018) 056004 [arXiv: 1711.01644] [INSPIRE].

[80] XENON collaboration, XENON1T Dark Matter Data Analysis: Signal Reconstruction, Calibration and Event Selection, Phys. Rev. D 100 (2019) 052014 [arXiv:1906.04717] [INSPIRE]. 